\title{
Well-posedness and persistence properties for two-component higher order Camassa-Holm systems with fractional inertia operator *
}

\author{
Rong Chen \\ College of Continuing Education, Chongqing Normal University, Chongqing 401331, PR China \\ Shouming Zhou ${ }^{\dagger}$ \\ College of Mathematics Science, Chongqing Normal University, Chongqing 401331, PR China
}

June 14, 2016

\begin{abstract}
In this paper, we study the Cauchy problem for a two-component higher order Camassa-Holm systems with fractional inertia operator $A=\left(1-\partial_{x}^{2}\right)^{r}, r \geq 1$, which was proposed by Escher and Lyons [20]. By the transport equation theory and Littlewood-Paley decomposition, we confirm the local well-posedness of solutions for the system in nonhomogeneous Besov spaces $B_{p, q}^{s} \times B_{p, q}^{s-2 r+1}$ with $1 \leq p, q \leq+\infty$ and the Besov index $s>\max \left\{2 r+\frac{1}{p}, 2 r+1-\frac{1}{p}\right\}$. Moreover, we demonstrate the local well-posedness in the critical Besov space $B_{2,1}^{2 r+\frac{1}{2}} \times B_{2,1}^{\frac{3}{2}}$. On the other hand, the propagation behaviour of compactly supported solutions is examined, namely whether solutions which are initially compactly supported will retain this property throughout their time of evolution. Finally, we also establish the persistence properties of the solutions to the two-component Camassa-Holm equation with $r=1$ in weighted $L_{\phi}^{p}:=L^{p}\left(\mathbb{R}, \phi^{p}(x) d x\right)$ spaces for a large class of moderate weights.

Keywords: Higher order Camassa-Holm systems, Well-posedness, Besov spaces, Infinite propagation speed, Persistence properties.

Mathematics Subject Classification(2000): 35G25, 35L05, 35Q50, 35Q53, 37K10.

*This work is supported by National Science Fund for Young Scholars of China (Grant No. 11301573), University Young Core Teacher Foundation of Chongqing, Technology Research Foundation of Chongqing Educational Committee (Grant No. KJ1400503), Natural Science Foundation of Chongqing (Grant No. cstc2014jcyjA00008), the Talent Project of Chongqing Normal University(Grant No. 14CSBJ05). The third author is supported by National Science Foundation of China (Grant No.11371384) and Natural Science Foundation of Chongqing (Grant N0. cstc2015jcyjBX0007).

${ }^{\dagger}$ E-mail: zhoushouming76@163.com
\end{abstract}

(C) 2016. This manuscript version is made available under the Elsevier user license http://www.elsevier.com/open-access/userlicense/1.0/ 


\section{Introduction}

In this paper, we consider the following Cauchy problem

$$
\left\{\begin{array}{lll}
m_{t}=\alpha u_{x}-b u_{x} m-u m_{x}-\kappa \rho \rho_{x}, & m=A u, & t>0, x \in \mathbb{R}, \\
\rho_{t}=-u \rho_{x}-(b-1) u_{x} \rho, & \alpha_{t}=0, & t>0, x \in \mathbb{R}, \\
u(x, 0)=u_{0}(x), & \rho(x, 0)=\rho_{0}(x), \quad t=0, x \in \mathbb{R},
\end{array}\right.
$$

where the inertia operator $A=\left(1-\partial_{x}^{2}\right)^{r}$ belongs to the class of fractional Sobolev norms $r \geq 1$, and the constants $b \in \mathbb{R}, \kappa \in \mathbb{R}$.

Obviously, if $\rho \equiv 0, A=1-\partial_{x}^{2}$ and $\alpha_{x}=0$ the Eq. (1.1) becomes a one-component family of equations which are parameterised by $b$ :

$$
u_{t}-u_{x x t}+\alpha u_{x}+(b+1) u u_{x}=b u_{x} u_{x x}+u u_{x x x}
$$

This family of so-called $b$-equations possess a number of structural phenomena which are shared by solutions of the family of equations (c.f.[21, 32,34]). By using Painlevé analysis, there are only two asymptotically integrable equations within this family: the Camassa-Holm $(\mathrm{CH})$ equation (Eq. (1.2) with $b=2$, c.f. $[4,5]$ ) and the Degasperis-Procesi (DP) equation (Eq. (1.2) with $b=3$, c.f. [14]). Integrable equations have widely been studied because they usually have very good properties including infinitely many conservation laws, infinite higher-order symmetries, bi-Hamiltonian structure, and Lax pair which make them solved by the inverse scattering method. Conserved quantities are very feasible for proving the existence of global solution in time, while a bi-Hamiltonian formulation helps in finding conserved quantities effectively. The advantage of the $\mathrm{CH}$ and $\mathrm{DP}$ equations in comparison to the $\mathrm{KdV}$ equation, lies in the fact that they not only have peaked solitons but also model the peculiar wave breaking phenomena (c.f. [5, 9]), and hence they represent the first examples of integrable equations which possess both global solutions and solutions which display wave-breaking in finite time, c.f. $[7,8,9]$.

In recent years, a number of integrable multi-component generalisations of the $\mathrm{CH}$ equation have been studied extensively. One of them is the following family of two-component system parameterised by $b$

$$
\left\{\begin{array}{l}
m_{t}=\alpha u_{x}-b u_{x} m-u m_{x}-\kappa \rho \rho_{x}, \quad m=u-u_{x x} \\
\rho_{t}=-u \rho_{x}-(b-1) u_{x} \rho, \quad \alpha, b \in \mathbb{R} .
\end{array}\right.
$$

Apparently, the two-component Camassa-Holm system [10], and the two-component Degasperis-Procesi system [30] are included in Eq. (1.3) as two special cases with $b=2$ and $b=3$, respectively. Recently, Escher et al. [19] presented the hydrodynamical derivation of the system (1.3), as a model for water waves with $\alpha$ a constant incorporating an underlying vorticity of the flow. They also proved the local well-posedness of (1.3) using a geometrical framework, studied the blow-up scenarios and global strong solutions of (1.3) on the circle.

All of these hydrodynamical models have a geometrical interpretation in terms of a geodesic flow on an appropriate infinite dimensional Lie group. The seminal work of Arnold [1] reformulated the Euler 
equation describing an ideal fluid, as a geodesic flow on the group of volume preserving diffeomorphisms of the fluid domain. Following this, Ebin and Marsden [18] reinterpreted this group of volume preserving diffeomorphisms as an inverse limit of Hilbert manifolds. This technique has been used in [11, 28] for the periodic $\mathrm{CH}$ equation. It was extended to (nonmetric) geodesic flows such as the DP equation in [22] and to right-invariant metrics induced by fractional Sobolev norm (non-local inertia operators) in [23]. In 2009, McLachlan and Zhang [27] studied the Cauchy problem for a modified CH equation derived as the Euler-Poincaré differential equation on the Bott-Virasoro group with respect to the $H^{k}$ metric, i.e.,

$$
m_{t}+2 u_{x} m+u m_{x}=0, \quad m=\left(1-\partial_{x}^{2}\right)^{k} u, \quad k \in \mathbb{N} .
$$

In [6], Coclite, Holden and Karlsen considered higher order Camassa-Holm equations (1.4) describing exponential curves of the manifold of smooth orientation-preserving diffeomorphisms of the unit circle in the plane. Recently, in [20], Escher and Lyons have shown that the system in equation (1.1) corresponds to a metric induced geodesic flow on the infinite dimensional Lie group Diff ${ }^{\infty}\left(\mathbb{S}^{1}\right) \mathbb{S} C^{\infty}\left(\mathbb{S}^{1}\right) \times \mathbb{R}$, where Diff ${ }^{\infty}\left(\mathbb{S}^{1}\right)$ denotes the group of orientation preserving diffeomorphisms of the circle, $C^{\infty}\left(\mathbb{S}^{1}\right)$ denotes the space of smooth functions on $\mathbb{S}^{1}$ while $(S)$ denotes an appropriate semi-direct product between the pair.

However, the Cauchy problem of (1.1) on the line has not been studied yet. In this paper, using the Littlewood-Paley theory, we established the local well-posedness of (1.1) in nonhomogeneous Besov spaces. Furthermore, inspired by the argument of D. Henry [25], we examine the propagation behaviour of compactly supported solutions, namely whether solutions which are initially compactly supported will retain this property throughout their time of evolution. In [24], the authors shown that the solution of Eq. (1.3) has exponential decay if the initial data in $H^{s} \times H^{s-1}$ with $s>5 / 2$ and has exponential decay, in present paper, working with moderate weight functions that are commonly used in time-frequency analysis [3], we generalize the persistence result on the solution to Eq. (1.3) in the weighted $L_{\phi}^{p}=L^{p}\left(\mathbb{R}, \phi^{p}(x) d x\right)$ spaces, and we also extend the Sobolev index to $s>3 / 2$.

Our paper is organized as follows. In Section 2, we establish the local well-posedness of the Cauchy problem associated with (1.1) in Besov spaces. In Section 3, we discuss the infinite propagation speed for (1.1). In the last section, we establish persistence properties and some unique continuation properties of the solutions to the Eq. (1.3) in weighted $L_{\phi}^{p}:=L^{p}\left(\mathbb{R}, \phi^{p}(x) d x\right)$ spaces.

\section{Local well-posedness in Besov spaces}

In this section, we shall discuss the local well-posedness of the Cauchy problem Eq. (1.1) in nonhomogeneous Besov spaces. The Littlewood-Paley theory and the properties of the Besov-Sobolev spaces referred to here may be found in $[2,12,24]$ and references therein.

\subsection{Local well-posedness in Besov spaces $B_{p, q}^{s}$}

In this section, we shall discuss the local well-posedness of the Cauchy problem (1.1). First, we present the following definition. 
Definition 2.1. For $T>0, s \in \mathbb{R}$ and $1 \leq p \leq+\infty$ and $s \neq 2+\frac{1}{p}$, we define

$$
\begin{gathered}
E_{p, q}^{s}(T) \doteq \mathcal{C}\left([0, T] ; B_{p, q}^{s}\right) \cap \mathcal{C}^{1}\left([0, T] ; B_{p, q}^{s-1}\right) \quad \text { if } r<+\infty \\
E_{p, \infty}^{s}(T) \doteq L^{\infty}\left([0, T] ; B_{p, \infty}^{s}\right) \cap \operatorname{Lip}\left([0, T] ; B_{p, \infty}^{s-1}\right),
\end{gathered}
$$

and $E_{p, q}^{s} \doteq \cap_{T>0} E_{p, q}^{s}(T)$.

The result of the local well-posedness in the Besov space is now stated as:

Theorem 2.1. Suppose that $1 \leq p, q \leq+\infty$ and $s>\max \left\{2 r+\frac{1}{p}, 2 r+1-\frac{1}{p}\right\}$ with $r \geq 1$. Let the function $\alpha(x) \equiv c \in \mathbb{R}$ or $\alpha(\cdot) \in B_{p, q}^{s-2 r}$, and the initial data $\left(u_{0}, \rho_{0}\right) \in B_{p, q}^{s} \times B_{p, q}^{s-2 r+1}$. Then there exists a time $T>0$ such that the Cauchy problem (1.1) has a unique solution $(u, \rho) \in E_{p, q}^{s}(T) \times E_{p, q}^{s-2 r+1}(T)$, and the map $\left(u_{0}, \rho_{0}\right) \mapsto(u, \rho)$ is continuous from a neighborhood of $\left(u_{0}, \rho_{0}\right)$ in $B_{p, q}^{s} \times B_{p, q}^{s-2 r+1}$ into

$$
\mathcal{C}\left([0, T] ; B_{p, q}^{s^{\prime}}\right) \cap \mathcal{C}^{1}\left([0, T] ; B_{p, q}^{s^{\prime}-1}\right) \times \mathcal{C}\left([0, T] ; B_{p, q}^{s^{\prime}-2 r+1}\right) \cap \mathcal{C}^{1}\left([0, T] ; B_{p, q}^{s^{\prime}-2 r}\right)
$$

$s^{\prime}<s$ when $q=+\infty$ whereas $s^{\prime}=s$ when $q<+\infty$.

Remark 2.1. When $p=q=2$, the Besov space $B_{p, q}^{s}$ coincides with the Sobolev space $H^{s}$. Thus under the condition $\left(u_{0}, \rho_{0}\right) \in H^{s} \times H^{s-2 r+1}$ with $s>2 r+\frac{1}{2}$ the above theorem implies that there exists a time $T>0$ such that the initial-value problem (1.1) has a unique solution $(u, \rho) \in \mathcal{C}\left([0, T] ; H^{s}\right) \cap \mathcal{C}^{1}\left([0, T] ; H^{s-1}\right) \times$ $\mathcal{C}\left([0, T] ; H^{s-2 r+1}\right) \cap \mathcal{C}^{1}\left([0, T] ; H^{s-2 r}\right)$, and the map $\left(u_{0}, \rho_{0}\right) \mapsto(u, \rho)$ is continuous from a neighborhood of $\left(u_{0}, \rho_{0}\right)$ in $H^{s} \times H^{s-2 r+1}$ into $\mathcal{C}\left([0, T] ; H^{s}\right) \cap \mathcal{C}^{1}\left([0, T] ; H^{s-1}\right) \times \mathcal{C}\left([0, T] ; H^{s-2 r+1}\right) \cap \mathcal{C}^{1}\left([0, T] ; H^{s-2 r}\right)$.

In the following, we denote $C>0$ a generic constant only depending on $p, q, s, b, \kappa, \mathcal{S}$ stands for the Schwartz space of smooth functions over $\mathbb{R}$ whose derivatives of all order decay at infinity. The set $\mathcal{S}^{\prime}$ of temperate distributions is the dual set of $\mathcal{S}$ for the usual pairing. Uniqueness and continuity with respect to the initial data are an immediate consequence of the following result.

Lemma 2.1. Let $1 \leq p, q \leq+\infty$ and $s>\max \left\{2 r+\frac{1}{p}, 2 r+1-\frac{1}{p}\right\}$ with $r \geq 1$, the function $\alpha(x) \equiv c \in \mathbb{R}$ or $\alpha(\cdot) \in B_{p, q}^{s-2 r}$. Suppose that $\left(u_{i}, \rho_{i}\right) \in\left\{L^{\infty}\left([0, T] ; B_{p, q}^{s}\right) \cap \mathcal{C}\left([0, T] ; \mathcal{S}^{\prime}\right)\right\} \times\left\{L^{\infty}\left([0, T] ; B_{p, q}^{s-2 r+1}\right) \cap \mathcal{C}\left([0, T] ; \mathcal{S}^{\prime}\right)\right\}$ $(i=1,2)$ be two given solutions of the initial-value problem (1.1) with the initial data $\left(u_{i}(0), \rho_{i}(0)\right) \in$ $B_{p, q}^{s} \times B_{p, q}^{s-2 r+1}(i=1,2)$, and denote $\rho_{12}=\rho_{1}-\rho_{2}, u_{12}=u_{1}-u_{2}$, i.e., $m_{12}=m_{1}-m_{2}$. Then for every $t \in[0, T]$, we have

(i) if $s>\max \left\{2 r+\frac{1}{p}, 2 r+1-\frac{1}{p}\right\}$ and $s \neq 2 r+2+\frac{1}{p}, s \neq 2 r+1+\frac{1}{p}$, then

$$
\left\|u_{12}\right\|_{B_{p, q}^{s-1}}+\left\|\rho_{12}\right\|_{B_{p, q}^{s-2 r}} \leq\left(\left\|u_{12}(0)\right\|_{B_{p, q}^{s-1}}+\left\|\rho_{12}(0)\right\|_{B_{p, q}^{s-2 r}}\right) \exp \left(C \int_{0}^{t} \Gamma_{s}(\tau) d \tau\right) .
$$

where

$$
\Gamma_{s}(t)=\left(\left\|u_{1}(t)\right\|_{B_{p, q}^{s}}+\left\|u_{2}(t)\right\|_{B_{p, q}^{s}}+\left\|\rho_{1}(t)\right\|_{B_{p, q}^{s-2 r+1}}+\left\|\rho_{2}(t)\right\|_{B_{p, q}^{s-2 r+1}}+\|\alpha\|\right),
$$

with

$$
\|\alpha\| \doteq \begin{cases}|c|, & \alpha(x) \equiv c \in \mathbb{R} \\ \|\alpha\|_{B_{p, q}^{s-2 r}}, & \alpha(\cdot) \in B_{p, q}^{s-2 r}\end{cases}
$$


(ii) if $s=2 r+2+1 / p$ or $s=2 r+1+1 / p$, then

$$
\left\|u_{12}\right\|_{B_{p, q}^{s-1}}+\left\|\rho_{12}\right\|_{B_{p, q}^{s-2 r}} \leq C\left(\left\|u_{12}(0)\right\|_{B_{p, q}^{s-1}}+\left\|\rho_{12}(0)\right\|_{B_{p, q}^{s-2 r}}\right)^{\theta} \exp \left(C \theta \int_{0}^{T} \Gamma_{s}(\tau) d \tau\right) \Gamma_{s}^{1-\theta}(t),
$$

where $\theta \in(0,1)$ and $\Gamma_{s}(t)$ as in case (i).

Proof. It is obvious that $u_{12} \in L^{\infty}\left([0, T] ; B_{p, q}^{s}\right) \cap \mathcal{C}\left([0, T] ; \mathcal{S}^{\prime}\right)$ and $\rho_{12} \in L^{\infty}\left([0, T] ; B_{p, q}^{s-2 r+1}\right) \cap \mathcal{C}\left([0, T] ; \mathcal{S}^{\prime}\right)$ which implies that $u_{12} \in \mathcal{C}\left([0, T] ; B_{p, q}^{s-1}\right), \rho_{12} \in \mathcal{C}\left([0, T] ; B_{p, q}^{s-2 r}\right)$. Moreover, if $\left(u_{i}, \rho_{i}\right)(i=1,2)$ are solution of Eq. (1.1) then it appears $u_{12}$ and $\rho_{12}$ are to satisfy the transport equations

$$
\begin{aligned}
& \partial_{t} m_{12}+u_{1} \partial_{x} m_{12}=-u_{12} \partial_{x} m_{2}-b m_{12} \partial_{x} u_{1}-b m_{2} \partial_{x} u_{12}+\alpha \partial_{x} u_{12}-\frac{\kappa}{2} \partial_{x}\left[\left(\rho_{1}+\rho_{2}\right) \rho_{12}\right], \\
& \partial_{t} \rho_{12}+u_{1} \partial_{x} \rho_{12}=-u_{12} \partial_{x} \rho_{2}-(b-1) \rho_{12} \partial_{x} u_{1}-(b-1) \rho_{2} \partial_{x} u_{12}, \\
& \left.u_{12}\right|_{t=0}=u_{12}(0) \doteq u_{1}(0)-u_{2}(0),\left.\rho_{12}\right|_{t=0}=\rho_{12}(0) \doteq \rho_{1}(0)-\rho_{2}(0), m_{12}=\left(1-\partial_{x}^{2}\right)^{r} u_{12} .
\end{aligned}
$$

Case I: $s>\max \left\{2 r+\frac{1}{p}, 2 r+1-\frac{1}{p}\right\}$ and $s \neq 2 r+2+\frac{1}{p}, s \neq 2 r+1+\frac{1}{p}$. According to Theorem 3.14 in [2], we have

$$
\begin{gathered}
\left\|m_{12}\right\|_{B_{p, q}^{s-2 r-1}} \leq\left\|m_{12}(0)\right\|_{B_{p, q}^{s-2 r-1}}+C \int_{0}^{t}\left(\left\|\partial_{x} u_{1}\right\|_{B_{p, q}^{s-2 r-2}}+\left\|\partial_{x} u_{1}\right\|_{B_{p, q}^{\frac{1}{p} \cap L^{\infty}}}\right)\left\|m_{12}\right\|_{B_{p, q}^{s-2 r-1}} d \tau \\
\quad+C \int_{0}^{t}\left\|u_{12} \partial_{x} m_{2}+b m_{12} \partial_{x} u_{1}+b m_{2} \partial_{x} u_{12}-\alpha \partial_{x} u_{12}+\frac{\kappa}{2} \partial_{x}\left[\left(\rho_{1}+\rho_{2}\right) \rho_{12}\right]\right\|_{B_{p, q}^{s-2 r-1}} d \tau .
\end{gathered}
$$

The condition $s>\max \left\{2 r+\frac{1}{p}, 2 r+1-\frac{1}{p}\right\} \geq 2 r+\frac{1}{p}$ with $r \geq 1$ yields that $B_{p, q}^{s-2 r}$ is an algebra, and we obtain

$$
\left\|\partial_{x} u_{1}\right\|_{B_{p, q}^{s-2 r-2}}+\left\|\partial_{x} u_{1}\right\|_{B_{p, q}^{\frac{1}{p}} \cap L^{\infty}} \leq 2\left\|\partial_{x} u_{1}\right\|_{B_{p, q}^{s-2 r}} \leq C\left\|u_{1}\right\|_{B_{p, q}^{s-r}}
$$

Note that the inertia operator $A=\left(1-\partial_{x}^{2}\right)^{r}$ be an $S^{2 r}$-multiplier, by Proposition 2.2 (7) in [34], we find that for all $s \in \mathbb{R}$

$$
\left\|u_{i}\right\|_{B_{p, q}^{s}} \approx\left\|m_{i}\right\|_{B_{p, q}^{s-2 r}}
$$

where the notation $f \approx g$ means that there exist two finite positive constants $C_{1}, C_{2}$ such that $C_{1} f \leq g \leq$ $C_{2} f$.

If $\max \left\{2 r+\frac{1}{p}, 2 r+1-\frac{1}{p}\right\}<s \leq 2 r+1+\frac{1}{p}$, by Morse-type estimate 2.5 (2) in [34] with $s_{1}=s-2 r-$ $1, s_{2}=s-2 r$, it is easy to check that $2 r+\frac{1}{2} \leq \max \left\{2 r+\frac{1}{p}, 2 r+1-\frac{1}{p}\right\}<s$, i.e., $s_{1}+s_{2}=2 s-4 r-1>0$. Recall that $B_{p, q}^{s-2 r}$ being an algebra for $s-2 r>\frac{1}{p}$ and the embedding $B_{p, q}^{s_{1}} \hookrightarrow B_{p, q}^{s_{2}}$ for $s_{1}<s_{2}$, we get

$$
\begin{aligned}
\| u_{12} \partial_{x} m_{2}+ & b m_{12} \partial_{x} u_{1}+b m_{2} \partial_{x} u_{12}-\alpha \partial_{x} u_{12}+\frac{\kappa}{2} \partial_{x}\left[\left(\rho_{1}+\rho_{2}\right) \rho_{12}\right] \|_{B_{p, q}^{s-2 r-1}} \\
\leq & C\left(\left\|u_{12}\right\|_{B_{p, q}^{s-2 r}}\left\|\partial_{x} m_{2}\right\|_{B_{p, q}^{s-2 r-1}}+\left\|m_{12}\right\|_{B_{p, q}^{s-2 r-1}}\left\|\partial_{x} u_{1}\right\|_{B_{p, q}^{s-2 r}}+\left\|m_{2}\right\|_{B_{p, q}^{s-2 r}}\left\|\partial_{x} u_{12}\right\|_{B_{p, q}^{s-2 r-1}}\right. \\
& \left.+\|\alpha\|\left\|u_{12}\right\|_{B_{p, q}^{s-2 r}}+\left\|\left(\rho_{1}+\rho_{2}\right) \rho_{12}\right\|_{B_{p, q}^{s-2 r}}\right) \\
\leq & C\left(\left\|u_{12}\right\|_{B_{p, q}^{s-2 r}}\left\|u_{2}\right\|_{B_{p, q}^{s}}+\left\|u_{12}\right\|_{B_{p, q}^{s-1}}\left\|u_{1}\right\|_{B_{p, q}^{s-2 r+1}}+\left\|u_{2}\right\|_{B_{p, q}^{s}}\left\|u_{12}\right\|_{B_{p, q}^{s-2 r}}\right. \\
& \left.+\|\alpha\|\left\|u_{12}\right\|_{B_{p, q}^{s-2 r}}+\left\|\left(\rho_{1}+\rho_{2}\right) \rho_{12}\right\|_{B_{p, q}^{s-2 r}}\right) \\
\leq & C\left(\left\|u_{1}\right\|_{B_{p, q}^{s}}+\left\|u_{2}\right\|_{B_{p, q}^{s}}+\|\alpha\|+\left\|\rho_{1}\right\|_{B_{p, q}^{s-2 r}}+\left\|\rho_{2}\right\|_{B_{p, q}^{s-2 r}}\right)\left(\left\|u_{12}\right\|_{B_{p, q}^{s-1}}+\left\|\rho_{12}\right\|_{B_{p, q}^{s-2 r}}\right) .
\end{aligned}
$$


For $s>2 r+1+\frac{1}{p}$, the above inequality also holds true in view of the fact that $B_{p, q}^{s-2 r-1}$ is an algebra. Thus, we may derive

$$
\left\|u_{12}\right\|_{B_{p, q}^{s-1}} \leq\left\|u_{12}(0)\right\|_{B_{p, q}^{s-1}}+C \int_{0}^{t}\left(\left\|u_{12}(\tau)\right\|_{B_{p, q}^{s-1}}+\left\|\rho_{12}(\tau)\right\|_{B_{p, q}^{s-2 r}}\right) \Gamma_{s}(\tau) d \tau .
$$

Similarly, we can also obtain the inequality for the component $\rho$ :

$$
\begin{aligned}
\left\|\rho_{12}\right\|_{B_{p, q}^{s-2 r}} \leq & \left\|\rho_{12}(0)\right\|_{B_{p, q}^{s-2 r}}+C \int_{0}^{t}\left(\left\|\partial_{x} u_{1}\right\|_{B_{p, q}^{s-2 r-1}}+\left\|\partial_{x} u_{1}\right\|_{B_{p, q}^{\frac{1}{p}} \cap L^{\infty}}\right)\left\|\rho_{12}\right\|_{B_{p, q}^{s-2 r}} d \tau \\
& +C \int_{0}^{t}\left\|u_{12} \partial_{x} \rho_{2}+(b-1) \rho_{12} \partial_{x} u_{1}+(b-1) \rho_{2} \partial_{x} u_{12}\right\|_{B_{p, q}^{s-2 r}} d \tau .
\end{aligned}
$$

The condition $s-2 r-1>\frac{1}{p}$ implies that

$$
\left\|\partial_{x} u_{1}\right\|_{B_{p, q}^{s-2 r-1}}+\left\|\partial_{x} u_{1}\right\|_{B_{p, q}^{\frac{1}{p}} \cap L^{\infty}} \leq 2\left\|\partial_{x} u_{1}\right\|_{B_{p, q}^{s-2 r-1}} \leq C\left\|u_{1}\right\|_{B_{p, q}^{s-2 r}} .
$$

If $2 r+\frac{1}{p}<s$, then $B_{p, q}^{s-2 r}$ being an algebra, we arrive at

$$
\begin{aligned}
\| u_{12} \partial_{x} \rho_{2} & +(b-1) \rho_{12} \partial_{x} u_{1}+(b-1) \rho_{2} \partial_{x} u_{12} \|_{B_{p, q}^{s-2 r}} \\
& \leq C\left(\left\|u_{12}\right\|_{B_{p, q}^{s-2 r}}\left\|\rho_{2}\right\|_{B_{p, q}^{s-2 r+1}}+\left\|\rho_{12}\right\|_{B_{p, q}^{s-2 r}}\left\|u_{1}\right\|_{B_{p, q}^{s-2 r+1}}+\left\|\rho_{2}\right\|_{B_{p, q}^{s-2 r}}\left\|u_{12}\right\|_{B_{p, q}^{s-2 r+1}}\right) \\
& \leq C\left\|u_{12}\right\|_{B_{p, q}^{s-1}}\left\|\rho_{2}\right\|_{B_{p, q}^{s-2 r+1}}+\left\|u_{1}\right\|_{B_{p, q}^{s-1}}\left\|\rho_{12}\right\|_{B_{p, q}^{s-2 r}}
\end{aligned}
$$

Combining (2.4)-(2.5), we get

$$
\left\|\rho_{12}\right\|_{B_{p, q}^{s-2 r}} \leq\left\|\rho_{12}(0)\right\|_{B_{p, q}^{s-2 r}}+C \int_{0}^{t}\left(\left\|u_{12}(\tau)\right\|_{B_{p, q}^{s-1}}+\left\|\rho_{12}(\tau)\right\|_{B_{p, q}^{s-2 r}}\right) \Gamma_{s}(\tau) d \tau .
$$

Therefore

$$
\left\|u_{12}\right\|_{B_{p, q}^{s-1}}+\left\|\rho_{12}\right\|_{B_{p, q}^{s-2 r}} \leq\left\|u_{12}(0)\right\|_{B_{p, q}^{s-1}}+\left\|\rho_{12}(0)\right\|_{B_{p, q}^{s-2 r}}+C \int_{0}^{t}\left(\left\|u_{12}\right\|_{B_{p, q}^{s-1}}+\left\|\rho_{12}\right\|_{B_{p, q}^{s-2 r}}\right) \Gamma_{s}(\tau) d \tau .
$$

Gronwall's lemma applied leads to (2.1).

Critical case II: $s=2 r+2+\frac{1}{p}$ or $s=2 r+1+\frac{1}{p}$. We only give the proof of $s=2 r+2+\frac{1}{p}$ by the interpolation method, and $s=2 r+1+\frac{1}{p}$ can be deal with as a similar approach. Indeed, if we choose $\theta=\frac{1}{2}\left(1-\frac{1}{2 p}\right) \in(0,1)$, then $2 r+1+\frac{1}{p}=\theta\left(2 r+\frac{1}{2 p}\right)+(1-\theta)\left(2 r+2+\frac{1}{2 p}\right)$ and $2+\frac{1}{p}=\theta\left(1+\frac{1}{2 p}\right)+(1-\theta)\left(3+\frac{1}{2 p}\right)$. According to the interpolation formula (Proposition 2.2(5) in [34]) and the obtained result (2.1), we have

$$
\begin{aligned}
& \left\|u_{12}\right\|_{B_{p, q}^{2 r+1+1 / p}}+\left\|\rho_{12}\right\|_{B_{p, q}^{2+1 / p}} \leq\left\|u_{12}\right\|_{B_{p, q}^{2 r+\frac{1}{2 p}}}^{\theta}\left\|u_{12}\right\|_{B_{p, q}^{2 r+2+\frac{1}{2 p}}}^{1-\theta}+\left\|\rho_{12}\right\|_{B_{p, q}^{1+\frac{1}{2 p}}}^{\theta}\left\|\rho_{12}\right\|_{B_{p, q}^{3+\frac{1}{2 p}}}^{1-\theta} \\
& \leq\left(\left\|u_{12}\right\|_{B_{p, q}^{2 r+\frac{1}{2 p}}}+\left\|\rho_{12}\right\|_{B_{p, q}^{1+\frac{1}{2 p}}}\right)^{\theta}\left(\left\|u_{12}\right\|_{\substack{2 r+2+\frac{1}{2 p} \\
B_{p, q}^{2 r}}}^{1-\theta}+\left\|\rho_{12}\right\|_{B_{p, q}^{3+\frac{1}{2 p}}}^{1-\theta}\right) \\
& \leq\left(\left\|u_{12}\right\|_{B_{p, q}^{2 r+\frac{1}{2 p}}}+\left\|\rho_{12}\right\|_{B_{p, q}^{1+\frac{1}{2 p}}}\right)^{\theta}\left(\left\|u_{1}\right\|_{B_{p, q}^{2 r+2+\frac{1}{2 p}}}+\left\|u_{2}\right\|_{B_{p, q}^{2 r+2+\frac{1}{2 p}}}+\left\|\rho_{1}\right\|_{B_{p, q}^{3+\frac{1}{2 p}}}+\left\|\rho_{2}\right\|_{B_{p, q}^{3+\frac{1}{2 p}}}\right)^{1-\theta} \\
& \leq C\left(\left\|u_{12}(0)\right\|_{B_{p, q}^{2 r+1+\frac{1}{p}}}+\left\|\rho_{12}(0)\right\|_{B_{p, q}^{2+\frac{1}{p}}}\right)^{\theta} \exp \left(C \theta \int_{0}^{T} \Gamma_{2 r+2+\frac{1}{p}}(\tau) d \tau\right) \Gamma_{2 r+2+\frac{1}{p}}^{1-\theta}(t),
\end{aligned}
$$

which yields the desired result.

Now, let us start the proof of Theorem 2.1, which is motivated by the proof of local existence theorem about Camassa-Holm type equations in $[12,34]$. Recall that $B_{p, q}^{\infty}=\cap_{s \in \mathbb{R}} B_{p, q}^{s}$ and the low frequency cutoff operator $S_{k+1}$ (c.f. $[12,34]$ ), by the classical Friedrichs regularization method, then we construct the approximate solutions to Eq. (1.1) as follows: 
Lemma 2.2. Let $p, q, r, s$ and $\alpha$ be as in the statement of Lemma 2.1. Assume that $u(0)=\rho(0):=0$. There exists a sequence of smooth functions $\left(u_{k}, \rho_{k}\right) \in \mathcal{C}\left(\mathbb{R}^{+} ; B_{p, q}^{\infty}\right)^{2}$ solving

$$
\left(T_{k}\right) \quad\left\{\begin{array}{l}
\partial_{t} m_{k+1}+u_{k} \partial_{x} m_{k+1}-\alpha u_{k}+b \partial_{x} u_{k} m_{k}+\kappa \rho_{k} \partial_{x} \rho_{k}=0, \\
\partial_{t} \rho_{k+1}+u_{k} \partial_{x} \rho_{k+1}+(b-1) \partial_{x} u_{k} \rho_{k}=0 \\
u_{k+1}(0)=S_{k+1} u(0), \quad \rho_{k+1}(0)=S_{k+1} \rho(0) .
\end{array}\right.
$$

Moreover, there is a positive time $T$ such that the solutions satisfy the following properties:

(i) $\left(u_{k}, \rho_{k}\right)_{k \in \mathbb{N}}$ is uniformly bounded in $E_{p, q}^{s}(T) \times E_{p, q}^{s-2 r+1}(T)$.

(ii) $\left(u_{k}, \rho_{k}\right)_{k \in \mathbb{N}}$ is a Cauchy sequence in $\mathcal{C}\left([0, T] ; B_{p, q}^{s-1}\right) \times \mathcal{C}\left([0, T] ; B_{p, q}^{s-2 r}\right)$.

Proof. Since all the data $S_{k+1} u(0)$ and $S_{k+1} \rho(0)$ belong to $B_{p, q}^{\infty}$, Lemma 2.3 in [34] indicates that for all $k \in \mathbb{N}$, the equation $\left(T_{k}\right)$ has a global solution in $\mathcal{C}\left(\mathbb{R}^{+} ; B_{p, q}^{\infty}\right)^{2}$.

For $s>\max \left\{2 r+\frac{1}{p}, 2 r+1-\frac{1}{p}\right\}$ and $s \neq 2 r+2+\frac{1}{p}$, thanks to Lemma 2.2 in [34] and the proof of Lemma 2.1, we have the following inequality for all $k \in \mathbb{N}$ :

$$
\begin{aligned}
& \left\|m_{k+1}\right\|_{B_{p, q}^{s-2 r}} \leq \exp \left(C \int_{0}^{t}\left(\left\|\partial_{x} u_{k}(\tau)\right\|_{B_{p, q}^{s-2 r-1}}+\left\|\partial_{x} u_{k}(\tau)\right\|_{B_{p, q}^{\frac{1}{p}} \cap L^{\infty}}\right) d \tau\right)\|m(0)\|_{B_{p, q}^{s-2 r}} \\
& +C \int_{0}^{t} \exp \left(C \int_{\tau}^{t}\left(\left\|\partial_{x} u_{k}\left(\tau^{\prime}\right)\right\|_{B_{p, q}^{s-2 r-1}}+\left\|\partial_{x} u_{k}\left(\tau^{\prime}\right)\right\|_{B_{p, q}^{\frac{1}{p}} \cap L^{\infty}}\right) d \tau^{\prime}\right)\left\|\alpha u_{k}-b \partial_{x} u_{k} m_{k}-\kappa \rho_{k} \partial_{x} \rho_{k}\right\|_{B_{p, q}^{s-2 r}} d \tau .
\end{aligned}
$$

and

$$
\begin{aligned}
\left\|\rho_{k+1}\right\|_{B_{p, q}^{s-2 r+1}} & \leq \exp \left(C \int_{0}^{t}\left(\left\|\partial_{x} u_{k}(\tau)\right\|_{B_{p, q}^{s-2 r}}+\left\|\partial_{x} u_{k}(\tau)\right\|_{B_{p}^{\frac{1}{p}} \cap q}\right) d \tau\right)\|\rho(0)\|_{B_{p, q}^{s-2 r+1}} \\
& +C \int_{0}^{t} \exp \left(C \int_{\tau}^{t}\left(\left\|\partial_{x} u_{k}\left(\tau^{\prime}\right)\right\|_{B_{p, q}^{s-2 r}}+\left\|\partial_{x} u_{k}\left(\tau^{\prime}\right)\right\|_{B_{p, q}^{\frac{1}{p}} \cap L^{\infty}}\right) d \tau^{\prime}\right)\left\|(b-1) \partial_{x} u_{k} \rho_{k}\right\|_{B_{p, q}^{s-2 r+1}} d \tau .
\end{aligned}
$$

Due to $s>2 r+\frac{1}{p}$, we know that $B_{p, q}^{s-2 r}$ is an algebra and $B_{p, q}^{s-2 r} \hookrightarrow L^{\infty}$. Furthermore, using the relationship $\|u\|_{B_{p, q}^{s}} \approx\|m\|_{B_{p, q}^{s-2 r}}$ implies

$$
\begin{aligned}
\left\|u_{k+1}\right\|_{B_{p, q}^{s}} & \leq \exp \left(2 C \int_{0}^{t}\left\|u_{k}(\tau)\right\|_{B_{p, q}^{s}} d \tau\right)\|u(0)\|_{B_{p, q}^{s}} \\
& +C \int_{0}^{t} \exp \left(2 C \int_{\tau}^{t}\left\|u_{k}\left(\tau^{\prime}\right)\right\|_{B_{p, q}^{s}} d \tau^{\prime}\right)\left(\|\alpha\|\left\|u_{k}\right\|_{B_{p, q}^{s-2 r}}+\left\|u_{k}\right\|_{B_{p, q}^{s-2 r+1}}\left\|u_{k}\right\|_{B_{p, q}^{s}}+\left\|\rho_{k}\right\|_{B_{p, q}^{s-2 r+1}}^{2}\right) d \tau .
\end{aligned}
$$

and

$$
\begin{aligned}
\left\|\rho_{k+1}\right\|_{B_{p, q}^{s-2 r+1}} & \leq \exp \left(2 C \int_{0}^{t}\left\|u_{k}(\tau)\right\|_{B_{p, q}^{s-2 r}} d \tau\right)\|\rho(0)\|_{B_{p, q}^{s-2 r+1}} \\
& +C \int_{0}^{t} \exp \left(2 C \int_{\tau}^{t}\left\|u_{k}\left(\tau^{\prime}\right)\right\|_{B_{p, q}^{s-2 r}} d \tau^{\prime}\right)\left\|u_{k}\right\|_{B_{p, q}^{s-2 r+2}}\left\|\rho_{k}\right\|_{B_{p, q}^{s-2 r+1}} d \tau .
\end{aligned}
$$

Thus, adding the two resulting inequalities yields

$$
\begin{aligned}
& \left\|u_{k+1}\right\|_{B_{p, q}^{s}}+\left\|\rho_{k+1}\right\|_{B_{p, q}^{s-2 r+1}} \leq \exp \left(2 C \int_{0}^{t}\left\|u_{k}(\tau)\right\|_{B_{p, q}^{s}} d \tau\right)\left(\|u(0)\|_{B_{p, q}^{s}}+\|\rho(0)\|_{B_{p, q}^{s-2 r+1}}\right) \\
& \quad+C \int_{0}^{t} \exp \left(2 C \int_{\tau}^{t}\left\|u_{k}\left(\tau^{\prime}\right)\right\|_{B_{p, q}^{s}} d \tau^{\prime}\right)\left(\|\alpha\|\left\|u_{k}\right\|_{B_{p, q}^{s}}+\left\|u_{k}\right\|_{B_{p, q}^{s}}^{2}+\left\|\rho_{k}\right\|_{B_{p, q}^{s-2 r+1}}^{2}+\left\|u_{k}\right\|_{B_{p, q}^{s}}\left\|\rho_{k}\right\|_{B_{p, q}^{s-2 r+1}}\right) d \tau \\
& \leq \exp \left(2 C \int_{0}^{t}\left\|u_{k}(\tau)\right\|_{B_{p, q}^{s}} d \tau\right)\left(\|u(0)\|_{B_{p, q}^{s}}+\|\rho(0)\|_{B_{p}^{s-2 r+1}}\right) \\
& \quad+C \int_{0}^{t} \exp \left(2 C \int_{\tau}^{t}\left\|u_{k}\left(\tau^{\prime}\right)\right\|_{B_{p, q}^{s}} d \tau^{\prime}\right)\left(\left\|u_{k}\right\|_{B_{p, q}^{s}}+\left\|\rho_{k}\right\|_{B_{p, q}^{s-2 r+1}}+\|\alpha\|\right)\left(\left\|u_{k}\right\|_{B_{p, q}^{s}}+\left\|\rho_{k}\right\|_{B_{p, q}^{s}-2 r+1}\right) d \tau .
\end{aligned}
$$


Hence if we define $Z_{k}(t)=\left\|u_{k}(t)\right\|_{B_{p, q}^{s}}+\left\|\rho_{k}(t)\right\|_{B_{p, q}^{s-2 r+1}}$ and $z_{0}=\|u(0)\|_{B_{p, q}^{s}}+\|\rho(0)\|_{B_{p, q}^{s-2 r+1}}$, then

$$
Z_{k+1}(t) \leq \exp \left(2 C \int_{0}^{t} Z_{k}(\tau) d \tau\right) z_{0}+C \int_{0}^{t} \exp \left(2 C \int_{\tau}^{t} Z_{k}\left(\tau^{\prime}\right) d \tau^{\prime}\right)\left(Z_{k}^{2}(\tau)+\|\alpha\| Z_{k}(\tau)\right) d \tau .
$$

Choosing

$$
T^{*}= \begin{cases}\min \left\{\frac{1}{8 C z_{0}}, \frac{1}{4 C\|\alpha\|}\right\}, & \text { for }\|\alpha\| \neq 0 \\ \frac{1}{8 C z_{0}}, & \text { for }\|\alpha\|=0\end{cases}
$$

Now, by induction, we show that for all $t \in\left[0, T^{*}\right)$

$$
Z_{k}(t) \leq \frac{2 z_{0}}{1-8 C z_{0} t}, \forall k \in \mathbb{N} .
$$

In fact, suppose (2.7) is valid for $k$, the condition $0 \leq \tau<t<T^{*} \leq \frac{1}{8 C\left(\left\|u_{0}\right\|_{B_{p}^{s}, q}+\left\|\rho_{0}\right\|_{B_{p}^{s, q}}^{s-2 r+1}\right)}$ implies

$$
\exp \left(2 C \int_{\tau}^{t} Z_{k}\left(\tau^{\prime}\right) d \tau^{\prime}\right) \leq \exp \left(\int_{\tau}^{t}\left(\frac{4 C z_{0}}{1-8 C z_{0} \tau^{\prime}}\right) d \tau^{\prime}\right)=\left(\frac{1-8 C z_{0} \tau}{1-8 C z_{0} t}\right)^{\frac{1}{2}}
$$

and

$$
\exp \left(2 C \int_{0}^{t} Z_{k}(\tau) d \tau\right) \leq\left(\frac{1}{1-8 C z_{0} t}\right)^{\frac{1}{2}}
$$

Inserting (2.7) into (2.6), we obtain

$$
\begin{aligned}
Z_{k+1}(t) & \leq \frac{z_{0}}{\sqrt{1-8 C z_{0} t}}+\frac{C}{\sqrt{1-8 C z_{0} t}} \int_{0}^{t}\left(\frac{4 z_{0}^{2}}{\left(1-8 C z_{0} \tau\right)^{\frac{3}{2}}}+\frac{2 z_{0}\|\alpha\|}{\left(1-8 C z_{0} \tau\right)^{\frac{1}{2}}}\right) d \tau \\
& =\frac{z_{0}}{1-8 C z_{0} t}+\frac{\|\alpha\|}{2 \sqrt{1-8 C z_{0} t}}\left(1-\sqrt{1-8 C z_{0} t}\right) \\
& =\frac{z_{0}}{1-8 C z_{0} t}+\frac{\|\alpha\|}{2\left(1-8 C z_{0} t\right)}\left(\sqrt{1-8 C z_{0} t}-1+8 C z_{0} t\right) \\
& \leq \frac{z_{0}}{1-8 C z_{0} t}+\frac{4 C\|\alpha\| z_{0} t}{\left(1-8 C z_{0} t\right)} \\
& \leq \frac{2 z_{0}}{1-8 C z_{0} t}
\end{aligned}
$$

in the last inequality we used that $t<T^{*} \leq \frac{1}{4 C\|\alpha\|}$ if $\|\alpha\| \neq 0$.

Now, we prove that $\left(u_{k}, \rho_{k}\right)_{k \in \mathbb{N}}$ is uniformly bounded in $\mathcal{C}\left([0, T] ; B_{p, q}^{s}\right) \times \mathcal{C}\left([0, T] ; B_{p, q}^{s-2 r+1}\right)$ for $T \in\left(0, T^{*}\right)$. Using the equation $\left(T_{k}\right)$ and the similar argument in the proof Lemma 2.1, one can easily prove that $\left(\partial_{t} u_{k}, \partial_{t} \rho_{k}\right)_{k \in \mathbb{N}}$ is uniformly bounded in $\mathcal{C}\left([0, T] ; B_{p, r}^{s-1}\right) \times \mathcal{C}\left([0, T] ; B_{p, r}^{s-2 r}\right)$, which yields that the sequence $\left(u_{k}, \rho_{k}\right)_{k \in \mathbb{N}}$ is uniformly bounded in $E_{p, r}^{s}(T) \times E_{p, r}^{s-2 r+1}(T)$.

Let us now show that $\left(u_{k}, \rho_{k}\right)_{k \in \mathbb{N}}$ is a Cauchy sequence in $\mathcal{C}\left([0, T] ; B_{p, q}^{s-1}\right) \times \mathcal{C}\left([0, T] ; B_{p, q}^{s-2 r}\right)$. In fact, for all $k, j \in \mathbb{N}$, from $\left(T_{k}\right)$, we have

$$
\left\{\begin{array}{l}
\left(\partial_{t}+u_{k+j} \partial_{x}\right)\left(m_{k+j+1}-m_{k+1}\right)=F(t, x) \\
\left(\partial_{t}+u_{k+j} \partial_{x}\right)\left(\rho_{k+j+1}-\rho_{k+1}\right)=G(t, x)
\end{array}\right.
$$

with

$$
\left\{\begin{aligned}
F(t, x)= & -\left(u_{k+j}-u_{k}\right) \partial_{x} m_{k+1}-b\left(m_{k+j}-m_{k}\right) \partial_{x} u_{k+j}-b m_{k} \partial_{x}\left(u_{k+j}-u_{k}\right) \\
& +\alpha \partial_{x}\left(u_{k+j}-u_{k}\right)-\frac{\kappa}{2} \partial_{x}\left[\left(\rho_{k+j}+\rho_{k}\right)\left(\rho_{k+j}-\rho_{k}\right)\right], \\
G(t, x)= & -\left(u_{k+j}-u_{k}\right) \partial_{x} \rho_{k+1}-(b-1)\left(\rho_{k+j}-\rho_{k}\right) \partial_{x} u_{k}-(b-1) \rho_{k} \partial_{x}\left(u_{k+j}-u_{k}\right) .
\end{aligned}\right.
$$


Applying Lemma 2.2 in [34] again, similar to the proof of Lemma 2.1, yields for every $t \in[0, T)$

$$
\begin{aligned}
\left\|u_{k+j+1}-u_{k+1}\right\|_{B_{p, q}^{s-1}} \leq & \exp \left(2 C \int_{0}^{t}\left\|u_{k+j}(\tau)\right\|_{B_{p, q}^{s}} d \tau\right)\left\|u_{k+j+1}(0)-u_{k+1}(0)\right\|_{B_{p, q}^{s-1}} \\
& +C \int_{0}^{t} \exp \left(2 C \int_{\tau}^{t}\left\|u_{k+j}\left(\tau^{\prime}\right)\right\|_{B_{p, q}^{s}} d \tau^{\prime}\right)\|F(\tau)\|_{B_{p, q}^{s-2 r-1}} d \tau,
\end{aligned}
$$

and

$$
\begin{aligned}
\left\|\rho_{k+j+1}-\rho_{k+1}\right\|_{B_{p, q}^{s-2 r}} & \leq \exp \left(2 C \int_{0}^{t}\left\|u_{k+j}(\tau)\right\|_{B_{p, q}^{s}} d \tau\right)\left\|\rho_{k+j+1}(0)-\rho_{k+1}(0)\right\|_{B_{p, q}^{s-2 r}} \\
& +C \int_{0}^{t} \exp \left(2 C \int_{\tau}^{t}\left\|u_{k+j}\left(\tau^{\prime}\right)\right\|_{B_{p, q}^{s}} d \tau^{\prime}\right)\|G(\tau)\|_{B_{p, q}^{s-2 r}} d \tau
\end{aligned}
$$

For $s>\max \left\{2 r+\frac{1}{p}, 2 r+1-\frac{1}{p}\right\}$ and $s \neq 2 r+2+\frac{1}{p}, 2 r+1+\frac{1}{p}$, we arrive at

$$
\|F(t)\|_{B_{p, q}^{s-2 r-1}}+\|G(t)\|_{B_{p, q}^{s-2 r}} \leq C\left(\left\|u_{k+j}-u_{k}\right\|_{B_{p, q}^{s-1}}+\left\|\rho_{k+j}-\rho_{k}\right\|_{B_{p, q}^{s-2 r}}\right) H(t),
$$

with

$$
H(t)=\left\|u_{k+1}\right\|_{B_{p, q}^{s}}+\left\|u_{k+j}\right\|_{B_{p, q}^{s}}+\left\|u_{k}\right\|_{B_{p, q}^{s}}+\|\alpha\|_{B_{p, q}^{s-2 r}}+\left\|\rho_{k+1}\right\|_{B_{p, q}^{s-2 r}}+\left\|\rho_{k+j}\right\|_{B_{p, q}^{s-2 r}}+\left\|\rho_{k}\right\|_{B_{p, q}^{s-2 r}}
$$

Submitting (2.10) inequality to (2.8)-(2.9), we get

$$
\begin{aligned}
V_{k+1}^{j}(t) \doteq & \left\|u_{k+j+1}-u_{k+1}\right\|_{B_{p, q}^{s-1}}+\left\|\rho_{k+j+1}-\rho_{k+1}\right\|_{B_{p, q}^{s-2 r}} \\
\leq & \exp \left(2 C \int_{0}^{t}\left\|u_{k+j}(\tau)\right\|_{B_{p, q}^{s}} d \tau\right)\left(\left\|u_{k+j+1}(0)-u_{k+1}(0)\right\|_{B_{p, q}^{s-1}}+\left\|\rho_{k+j+1}(0)-\rho_{k+1}(0)\right\|_{B_{p, q}^{s-2 r}}\right. \\
& \left.+C \int_{0}^{t} \exp \left(2 C \int_{\tau}^{t}\left\|u_{k+j}\left(\tau^{\prime}\right)\right\|_{B_{p, q}^{s}} d \tau^{\prime}\right)\left(\|F(\tau)\|_{B_{p, q}^{s-2 r-1}}+\|G(\tau)\|_{B_{p}^{s-2 r-1}}\right) d \tau\right) \\
\leq & \exp \left(2 C \int_{0}^{t}\left\|u_{k+j}(\tau)\right\|_{B_{p, q}^{s}} d \tau\right)\left(V_{k+1}^{j}(0)+C \int_{0}^{t} \exp \left(-2 C \int_{0}^{\tau}\left\|u_{k+j}\left(\tau^{\prime}\right)\right\|_{B_{p, q}^{s}} d \tau^{\prime}\right) V_{k}^{j}(\tau) H(\tau) d \tau\right) .
\end{aligned}
$$

As per Proposition 2.1 in [34], we have

$$
\begin{aligned}
\left\|u_{k+j+1}(0)-u_{k+1}(0)\right\|_{B_{p, q}^{s-1}} & =\left\|S_{k+j+1} u(0)-S_{k+1} u(0)\right\|_{B_{p, q}^{s-1}}=\left\|\sum_{d=k+1}^{k+j} \Delta_{d} u_{0}\right\|_{B_{p, q}^{s-1}} \\
& =\left(\sum_{k \geq-1} 2^{k(s-1) \sigma}\left\|\Delta_{k}\left(\sum_{d=k+1}^{k+j} \Delta_{d} u_{0}\right)\right\|_{L^{p}}^{\sigma}\right)^{\frac{1}{\sigma}} \\
& \leq C\left(\sum_{d=k}^{k+j+1} 2^{-d \sigma} 2^{d \sigma s}\left\|\Delta_{d} u_{0}\right\|_{L^{p}}^{\sigma}\right)^{\frac{1}{\sigma}} \leq C 2^{-k}\left\|u_{0}\right\|_{B_{p, q}^{s}} .
\end{aligned}
$$

Similarly, we obtain

$$
\left\|\rho_{k+j+1}(0)-\rho_{k+1}(0)\right\|_{B_{p, q}^{s-2 r}} \leq C 2^{-k}\left\|\rho_{0}\right\|_{B_{p, q}^{s-2 r+1}} .
$$

Due to $\left\{u_{k}, \rho_{k}\right\}_{k \in \mathbb{N}}$ being uniformly bounded in $E_{p, q}^{s}(T) \times E_{p, q}^{s-2 r+1}(T)$, one may find a positive constant $C_{T}$ independent of $k, j$ such that

$$
V_{k+1}^{j}(t) \leq C_{T}\left(2^{-k}+\int_{0}^{t} V_{k}^{j}(\tau) d \tau\right), \forall t \in[0, T] .
$$

Employing the induction procedure with respect to the index $k$ leads to

$$
V_{k+1}^{j}(t) \leq C_{T}\left(2^{-k} \sum_{i=0}^{k} \frac{\left(2 T C_{T}\right)^{i}}{i !}+C_{T}^{k+1} \int \frac{(t-\tau)^{k}}{k !} d \tau\right) \leq 2^{-k}\left(C_{T} \sum_{i=0}^{k} \frac{\left(2 T C_{T}\right)^{i}}{i !}\right)+C_{T} \frac{\left(T C_{T}\right)^{k+1}}{(k+1) !}
$$


which reveals the desired result as $k \rightarrow \infty$. Finally, applying the interpolation method to the critical case $s=2 r+2+\frac{1}{p}$ concludes the proof of Lemma 2.2 .

Finally, we prove the existence and uniqueness for Eq. (1.1) in Besov space.

Proof of Theorem 2.1. In view of Lemma 2.2, we have that the pair $\left(u_{k}, \rho_{k}\right)_{k \in \mathbb{N}}$ is a Cauchy sequence in $\mathcal{C}\left([0, T] ; B_{p, q}^{s-1}\right) \times \mathcal{C}\left([0, T] ; B_{p, q}^{s-2 r}\right)$, thus, it converges to some limit function $(u, \rho) \in \mathcal{C}\left([0, T] ; B_{p, q}^{s-1}\right) \times$ $\mathcal{C}\left([0, T] ; B_{p, q}^{s-2 r}\right)$. Next, we show that $(u, \rho) \in E_{p, q}^{s}(T) \times E_{p, q}^{s-2 r}(T)$ solves system (1.1). According to Lemma 2.2 , one can see that $\left(u_{k}, \rho_{k}\right)_{k \in \mathbb{N}}$ is uniformly bounded in $L^{\infty}\left([0, T] ; B_{p, q}^{s}\right) \times L^{\infty}\left([0, T] ; B_{p, q}^{s-2 r+1}\right)$. Fatou property for Besov spaces (Proposition 2.2(6) in [34]) guarantees that $(u, \rho)$ also belong to $L^{\infty}\left([0, T] ; B_{p, q}^{s}\right) \times$ $L^{\infty}\left([0, T] ; B_{p, q}^{s-2 r+1}\right)$.

On the other hand, as $\left(u_{k}, \rho_{k}\right)_{k \in \mathbb{N}}$ converges to $(u, \rho)$ in $\mathcal{C}\left([0, T] ; B_{p, q}^{s-1}\right) \times \mathcal{C}\left([0, T] ; B_{p, q}^{s-2 r}\right)$, an interpolation argument insures that the convergence holds in $\mathcal{C}\left([0, T] ; B_{p, q}^{s^{\prime}}\right) \times \mathcal{C}\left([0, T] ; B_{p, q}^{s^{\prime}-2 r+1}\right)$, for any $s^{\prime}<s$.

Taking limit in $T_{k}$ reveals that $(u, \rho)$ satisfy system $(1.1)$ in the sense of $\mathcal{C}\left([0, T] ; B_{p, q}^{s^{\prime}-1}\right) \times \mathcal{C}\left([0, T] ; B_{p, q}^{s^{\prime}-2 r}\right)$ for all $s^{\prime}<s$. In view of the fact that $(u, \rho)$ belongs to $L^{\infty}\left([0, T] ; B_{p, q}^{s}\right) \times L^{\infty}\left([0, T] ; B_{p, q}^{s-2 r+1}\right)$ and $B_{p, q}^{s}$ is an algebra as $s>2 r+\frac{1}{p}$, we get that the right-hand side of the equation

$$
m_{t}+u m_{x}=\alpha u_{x}-b u_{x} m-\kappa \rho \rho_{x}
$$

belongs to $L^{\infty}\left([0, T] ; B_{p, q}^{s}\right)$, and the right-hand side of the second equation

$$
\rho_{t}+u \rho_{x}=-(b-1) u_{x} \rho
$$

belongs to $L^{\infty}\left([0, T] ; B_{p, q}^{s-2 r+1}\right)$. In particular, for the case $q<\infty$, Lemma 2.2 yields that $(u, \rho) \in \mathcal{C}\left([0, T] ; B_{p, q}^{s^{\prime}}\right) \times$ $\mathcal{C}\left([0, T] ; B_{p, q}^{s^{\prime}-2 r+1}\right)$ for any $s^{\prime}<s$. Finally, applying the equation again, we see that $\left(\partial_{t} u, \partial_{t} \rho\right) \in \mathcal{C}\left([0, T] ; B_{p, q}^{s-1}\right) \times$ $\mathcal{C}\left([0, T] ; B_{p, q}^{s-2 r}\right)$ if $q<\infty$, and in $L^{\infty}\left([0, T] ; B_{p, q}^{s-1}\right) \times L^{\infty}\left([0, T] ; B_{p, q}^{s-2 r}\right)$ otherwise. Therefore, the pair $(u, v) \in E_{p, q}^{s}(T) \times E_{p, q}^{s-2 r+1}(T)$.

On the other hand, the continuity with respect to the initial data in

$$
\mathcal{C}\left([0, T] ; B_{p, q}^{s^{\prime}}\right) \cap \mathcal{C}^{1}\left([0, T] ; B_{p, q}^{s^{\prime}-1}\right) \times \mathcal{C}\left([0, T] ; B_{p, q}^{s^{\prime}-2 r+1}\right) \cap \mathcal{C}^{1}\left([0, T] ; B_{p, q}^{s^{\prime}-2 r}\right) \quad \text { for all } s^{\prime}<s,
$$

can be obtained by Lemma 2.1 and a simple interpolation argument. Motivated by [13], the case $s^{\prime}=s$ can be proved through the use of a sequence of viscosity approximation solutions $\left(u_{\epsilon}, \rho_{\epsilon}\right)_{\epsilon>0}$ for System (1.1) which converges uniformly in

$$
\mathcal{C}\left([0, T] ; B_{p, q}^{s}\right) \cap \mathcal{C}^{1}\left([0, T] ; B_{p, q}^{s-1}\right) \times \mathcal{C}\left([0, T] ; B_{p, q}^{s-2 r+1}\right) \cap \mathcal{C}^{1}\left([0, T] ; B_{p, q}^{s-2 r}\right)
$$

gives the continuity of solution $(u, \rho) \in E_{p, q}^{s}(T) \times E_{p, q}^{s-2 r+1}(T)$.

\subsection{Critical case}

It is well known that $B_{2,2}^{s}(\mathbb{R})=H^{s}$ and for any $s^{\prime}<2 r+\frac{1}{2}<s$ :

$$
H^{s} \hookrightarrow B_{2,1}^{2 r+\frac{1}{2}} \hookrightarrow H^{2 r+\frac{1}{2}} \hookrightarrow B_{2, \infty}^{2 r+\frac{1}{2}} \hookrightarrow H^{s^{\prime}}
$$

which shows that $H^{s}$ and $B_{2,1}^{s}$ are quite close. So, attention is now restricted of critical case in the local well-posedness. 
Theorem 2.2. Suppose that the function $\alpha(x) \equiv c \in \mathbb{R}$ or $\alpha(\cdot) \in B_{2,1}^{1 / 2}$, and the initial data $z_{0} \doteq\left(u_{0}, \rho_{0}\right) \in$ $B_{2,1}^{2 r+\frac{1}{2}} \times B_{2,1}^{\frac{3}{2}}$. Then there exists a time $T>0$ such that the Cauchy problem (1.1) has a unique solution

$$
z=z\left(\cdot, z_{0}\right) \in \mathcal{C}\left([0, T] ; B_{2,1}^{2 r+\frac{1}{2}}\right) \cap \mathcal{C}^{1}\left([0, T] ; B_{2,1}^{2 r-\frac{1}{2}}\right) \times \mathcal{C}\left([0, T] ; B_{2,1}^{\frac{3}{2}}\right) \cap \mathcal{C}^{1}\left([0, T] ; B_{2,1}^{\frac{1}{2}}\right)
$$

Moreover, the solution depends continuously on the initial data, i.e. the mapping

$$
z_{0} \mapsto z\left(\cdot, z_{0}\right): B_{2,1}^{2 r+\frac{1}{2}} \times B_{2,1}^{\frac{3}{2}} \mapsto \mathcal{C}\left([0, T] ; B_{2,1}^{2 r+\frac{1}{2}}\right) \cap \mathcal{C}^{1}\left([0, T] ; B_{2,1}^{2 r-\frac{1}{2}}\right) \times \mathcal{C}\left([0, T] ; B_{2,1}^{\frac{3}{2}}\right) \cap \mathcal{C}^{1}\left([0, T] ; B_{2,1}^{\frac{1}{2}}\right)
$$

is continuous.

Proof. On account of $\left(u_{0}, \rho_{0}\right) \in B_{2,1}^{2 r+\frac{1}{2}} \times B_{2,1}^{\frac{3}{2}}$, the transport theory (see Lemma 2.2 in [34]) can be applied. Similar to the case $\left(u_{0}, \rho_{0}\right) \in B_{p, q}^{s} \times B_{p, q}^{s-2 r+1}$ with $s>\max \left\{2 r+\frac{1}{p}, 2 r+1-\frac{1}{p}\right\}$ and the proof of Theorem 1 in [12], we can establish this result. The proof of the theorem is therefore omitted without details.

\section{Compact support}

Next, let us consider the following initial value problem:

$$
\begin{cases}\varphi_{t}=u(t, \varphi(t, x)), & t \in[0, T), \quad x \in \mathbb{R}, \\ \varphi(0, x)=x, & t=0, \quad x \in \mathbb{R},\end{cases}
$$

where $u$ is the first component of the solution $(u, \rho)$ for the problem (1.1).

Lemma 3.1. (see [33]) Let $\left(u_{0}, \rho_{0}\right) \in H^{s} \times H^{s-2 r+1}$ with $s>2 r+1 / 2$, and $T>0$ be the life span of the solution to Eq. (1.1). Then there exists a unique solution $\varphi \in \mathcal{C}^{1}([0, T), \mathbb{R})$ to Eq. (3.1). Moreover, the map $\varphi(t, \cdot)$ is an increasing diffeomorphism over $\mathbb{R}$, where

$$
\varphi_{x}(t, x)=\exp \left\{\int_{0}^{t} u_{\varphi}(s, \varphi(s, x)) d s\right\}>0,
$$

for all $(t, x) \in[0, T) \times \mathbb{R}$.

Our next result shows the component $\rho$ of the solution for system (1.1) retains the property of being compactly supported as it evolves over time, totally independent of the form of the initial data $u_{0}$ (through $\left.m_{0}\right)$. In the following we assume that $\left(u_{0}, \rho_{0}\right) \in H^{s} \times H^{s-2 r+1}$ with $s>2 r+1 / 2$, and so $\rho$ is a classical solution of the system.

Lemma 3.2. Let $\left(u_{0}, \rho_{0}\right) \in H^{s} \times H^{s-2 r+1}$ with $s>2 r+1 / 2$, and $T>0$ be the maximal existence time of the corresponding solution $(u, \rho)$ to system (1.1). Then, we have

$$
\rho(t, \varphi(t, x)) \varphi_{x}^{b-1}=\rho(0, x)
$$

Moreover, if there exist $M_{1}>0$ such that $(b-1) u_{\varphi}(t, \varphi) \geq-M_{1}$ for all $(t, x) \in[0, T) \times \mathbb{R}$, then

$$
\|\rho(t, \cdot)\|_{L^{\infty}}=\|\rho(t, \varphi(t, \cdot))\|_{L^{\infty}} \leq \exp \left\{M_{1} T\right\}\left\|\rho_{0}(\cdot)\right\|_{L^{\infty}}, \quad \text { for all } t \in[0, T)
$$

Furthermore, if $\int_{\mathbb{R}}\left|\rho_{0}(x)\right|^{\frac{1}{b-1}} d x$ converge with $b \neq 1$, then

$$
\int_{\mathbb{R}}|\rho(t, x)|^{\frac{1}{b-1}} d x=\int_{\mathbb{R}}\left|\rho_{0}(x)\right|^{\frac{1}{b-1}} d x, \text { for all } t \in[0, T),
$$


Proof. Noticing $\frac{d \varphi_{x}(t, x)}{d t}=\varphi_{x t}=u_{\varphi}(t, \varphi(t, x)) \varphi_{x}(t, x)$, differentiating the left-hand side of the equation in (3.3) with respect to $t$, and using the second equation in (1.1), we obtain

$$
\begin{aligned}
\frac{d}{d t}\left(\rho(t, \varphi(t, x)) \varphi_{x}^{b-1}\right) & =\rho_{t}(t, \varphi(t, x)) \varphi_{x}^{b-1}+\rho_{\varphi}(t, \varphi(t, x)) \varphi_{t} \varphi_{x}^{b-1}+(b-1) \rho(t, \varphi(t, x)) \varphi_{x}^{b-2} \varphi_{x t} \\
& =\varphi_{x}^{b-1}\left(\rho_{t}(t, \varphi(t, x))+\rho_{\varphi}(t, \varphi(t, x)) u(t, \varphi(t, x))+(b-1) \rho(t, \varphi(t, x)) u_{\varphi}(t, \varphi(t, x))\right) \\
& =0 .
\end{aligned}
$$

which means that $\rho(t, \varphi(t, x)) \varphi_{x}^{b-1}$ is independent on the time $t$. By $(3.1)$, we know $\varphi(0, x)=x$ and $\varphi_{x}(x, 0)=1$, thus, Eq. (3.3) holds.

By Lemma 3.1, in view of Eq. (3.3), and $\varphi_{x}(0, x)=1$ we have

$$
\begin{aligned}
\|\rho(t, \cdot)\|_{L^{\infty}} & =\|\rho(t, \varphi(t, \cdot))\|_{L^{\infty}}=\left\|\varphi_{x}^{1-b} \rho_{0}\right\|_{L^{\infty}} \\
& =\left\|\exp \left\{(1-b) \int_{0}^{t} u_{\varphi}(s, \varphi(s, x)) d s\right\} \rho_{0}(\cdot)\right\|_{L^{\infty}} \\
& \leq \exp \left\{M_{1} T\right\}\left\|\rho_{0}(\cdot)\right\|_{L^{\infty}} \quad \text { for all } t \in[0, T),
\end{aligned}
$$

and

$$
\begin{aligned}
\int_{\mathbb{R}}\left|\rho_{0}(x)\right|^{\frac{1}{b-1}} d x & =\int_{\mathbb{R}}|\rho(t, \varphi(t, x))|^{\frac{1}{b-1}} \varphi_{x}(t, x) d x=\int_{\mathbb{R}}|\rho(t, \varphi(t, x))|^{\frac{1}{b-1}} d \varphi(t, x) \\
& =\int_{\mathbb{R}}|\rho(t, x)|^{\frac{1}{b-1}} d x \text { for all } t \in[0, T)
\end{aligned}
$$

which guarantee the lemma is true.

Corollary 3.1. If $\rho(t, x)$ is a solution for the second equation in (1.1) with initial data $\rho_{0}=\rho(0, x)$ then we have the following representation,

$$
\rho(t, x)=\rho_{0}\left(\varphi^{-1}(t, x)\right) e^{(1-b) \int_{0}^{t} u_{x}(s, x) d s}, \quad t \in[0, T) .
$$

Moreover, if $\rho_{0}=\rho(0, x)$ is supported in the interval $\left[\beta_{\rho_{0}}, \gamma_{\rho_{0}}\right]$, then $\rho$ will remain compactly supported for all further times of its existence $t \in[0, T)$, with the support of $\rho(t, x)$ contained in the interval $\left[\varphi\left(t, \beta_{\rho_{0}}\right), \varphi\left(t, \gamma_{\rho_{0}}\right)\right]$.

Proof. The relation (3.5) follows from the identities (3.3) and (3.2) and the invertibility of $\varphi$ for each $t \in[0, T)$. Furthermore, since $\varphi_{x}>0$, and $\rho_{0}$ is compactly supported, it follows from this relation that $\rho(t, x)$ is compactly supported for all times $t \in[0, T)$, with support contained in $\left[\varphi\left(t, \beta_{\rho_{0}}\right), \varphi\left(t, \gamma_{\rho_{0}}\right)\right]$.

The next result proves a similar property for solutions $m$ of (1.1), namely that a solution which is initially compactly supported retains this property throughout its evolution. However, whereas Corollary 3.1 made no assumptions on the form of the initial data $u_{0}, m_{0}$ aside from the required level of smoothness, Corollary 3.2 below requires that $\rho_{0}$ be also compactly supported.

Corollary 3.2. Assume that $u_{0}$ is such that $m_{0}=A u_{0}$ has compact support, contained in the interval $\left[\beta_{m_{0}}, \gamma_{m_{0}}\right]$, and that $\rho_{0}$ is also compactly supported, with support contained in $\left[\beta_{\rho_{0}}, \gamma_{\rho_{0}}\right]$. If $T=T\left(u_{0}, \rho_{0}\right)>0$ is the maximal existence time of the unique classical solutions $u(t, x), \rho(t, x)$ to the system of equations (1.1) with the given initial data $\left(u_{0}(x), \rho_{0}(x)\right)$ and $\alpha \equiv 0$, then for any $t \in[0, T)$ the $C^{1}$ function $x \mapsto m(t, x)$ has compact support. 
Proof. Applying the family of diffeomorphisms (3.1) and the first equation in (1.1), we get

$$
\begin{aligned}
\frac{d}{d t} & \left(m(t, \varphi(t, x)) \varphi_{x}^{b}(t, x)\right) \\
& =m_{t}(t, \varphi(t, x)) \varphi_{x}^{b}+m_{\varphi}(t, \varphi(t, x)) \varphi_{t} \varphi_{x}^{b}+b m(t, \varphi(t, x)) \varphi_{x}^{b-1} \varphi_{x t} \\
& =\varphi_{x}^{b}\left(m_{t}(t, \varphi(t, x))+m_{\varphi}(t, \varphi(t, x)) \varphi_{t}+b m(t, \varphi(t, x)) u_{\varphi}(t, \varphi(t, x))\right) \\
& =-\kappa \varphi_{x}^{b}(t, x) \rho(t, \varphi(t, x)) \rho_{\varphi}(t, \varphi(t, x))
\end{aligned}
$$

Therefore

$$
m(t, \varphi(t, x)) \varphi_{x}^{b}(t, x)=m_{0}(x)-\kappa \int_{0}^{t} \rho(s, \varphi(t, x)) \rho_{\varphi}(s, \varphi(t, x)) \varphi_{x}^{b}(s, x) d s
$$

Now, by assumption, $m_{0}(x)$ is supported in the compact interval $\left[\beta_{m_{0}}, \gamma_{m_{0}}\right]$. For fixed $t \in[0, T)$, Corollary 3.1 assures us that $\rho(t, \cdot)$ is compactly supported in the interval $\left[\beta_{\rho_{0}}, \gamma_{\rho_{0}}\right]$, and therefore the integral term in (3.6), regarded as a function of $x$, must also be compactly supported in the interval $\left[\beta_{\rho_{0}}, \gamma_{\rho_{0}}\right]$. Therefore, setting $\beta=\max \left\{\beta_{m_{0}}, \beta_{\rho_{0}}\right\}, \gamma=\min \left\{\gamma_{m_{0}}, \gamma_{\rho_{0}}\right\}$, it follows that $m(t, \cdot)$ is compactly supported, with its support contained in the interval $[\varphi(t, \beta), \varphi(t, \gamma)]$, for all $t \in[0, T)$.

\section{On the Persistence Properties of the Eq. (1.3)}

In Section 3, we examined the propagation behaviour of compactly supported solutions, namely the solutions which are initially compactly supported will retain this property throughout their time of evolution. In [24], the authors shown that the solutions possess exponentially decaying profiles for large values of the spatial variable. Motivated by the recent works $[3,31]$ on the nonlinear Camassa-Holm equation in weighted Sobolev spaces. The aim of this section is to establish the persistence properties for the Eq. (1.3) in weighted $L^{p}$ spaces. Our results generalize the work presented in [24] on infinite propagation speed and asymptotic profiles to Eq. (1.3) in $L_{\phi}^{p}$.

In the present section, we intend to find a large class of weight functions $\phi$ such that

$$
\sup _{t \in[0, T)}\left(\|u(t) \phi\|_{L^{p}}+\left\|u_{x}(t) \phi\right\|_{L^{p}}+\|\rho(t) \phi\|_{L^{p}}\right)<\infty
$$

this way we obtain a persistence result on solutions $(u, \rho)$ to Eq. (1.3) in the weight $L^{p}$ spaces $L_{\phi}^{p}:=$ $L^{p}\left(\mathbb{R}, \phi^{p}(x) d x\right)$. As a consequence and an application we determine the spatial asymptotic behavior of certain solutions to Eq. (1.3). We will work with moderate weight functions which appear with regularity in the theory of time-frequency analysis and have led to optimal results for the Camassa-Holm equation in [3], we first give the definition for admissible weight function. The pre-defined terminologies like $v$-moderate, sub-multiplicative can be refer the reader to $[3,31])$.

Definition 4.1. An admissible weight function for the Eq. (1.3) is a locally absolutely continuous function $\phi: \mathbb{R} \rightarrow \mathbb{R}$ such that, for some $A>0$ and almost all $x \in \mathbb{R},\left|\phi^{\prime}(x)\right| \leq A|\phi(x)|$, and that is $v$-moderate for some sub-multiplicative weight function $v$ satisfying $\inf _{\mathbb{R}} v>0$ and

$$
\int_{\mathbb{R}} \frac{v(x)}{e^{|x|}} d x<\infty
$$


We can now state our main result on admissible weights.

Theorem 4.1. Assume that $u_{0} \phi, u_{0, x} \phi, \rho_{0} \phi \in L^{p}(\mathbb{R}), 1 \leq p \leq \infty$ for an admissible weight function $\phi$ of Eq. (1.3), and $T$ be the maximal time of the solution $z=(u, \rho)$ to system (1.3) with the initial data $z_{0}=\left(u_{0}, \rho_{0}\right) \in H^{s}(\mathbb{R}) \times H^{s-1}(\mathbb{R}), s>3 / 2$. Then, for all $t \in[0, T]$, there is a constant $C>0$ depending only on the weight $\phi$ such that

$$
\|u(t) \phi\|_{L^{p}}+\left\|u_{x}(t) \phi\right\|_{L^{p}}+\|\rho(t) \phi\|_{L^{p}} \leq\left(\left\|u_{0} \phi\right\|_{L^{p}}+\left\|u_{0, x} \phi\right\|_{L^{p}}+\left\|\rho_{0} \phi\right\|_{L^{p}}\right) \exp \{C(1+M) t\},
$$

where

$$
M \doteq \sup _{t \in[0, T]}\left(\|u(t)\|_{L^{\infty}}+\left\|u_{x}(t)\right\|_{L^{\infty}}+\|\rho(t)\|_{L^{\infty}}\right)<\infty
$$

If we take the standard weights $\phi=\phi_{a, b, c, d}(x)=e^{a|x|^{b}}(1+|x|)^{c} \log (e+|x|)^{d}$ with the following conditions:

$$
a \geq 0, \quad c, d \in \mathbb{R}, \quad 0 \leq b \leq 1, a b<1
$$

then, the restriction $a b<1$ guarantees the validity of condition (4.1) for a multiplicative function $v(x) \geq 1$. Thus, the classical example of the application for Theorem 4.1 is the following special persistence properties.

Remark 4.1. (1) Take $\phi=\phi_{0,0, c, 0}$ with $c>0$, and choose $p=\infty$. In this case the Theorem 4.1 states that the condition

$$
\left|u_{0}(x)\right|+\left|u_{0, x}(x)\right|+\left|\rho_{0}(x)\right| \leq C(1+|x|)^{-c}
$$

implies the uniform algebraic decay in $[0, T)$ :

$$
|u(x, t)|+\left|u_{x}(x, t)\right|+|\rho(x, t)| \leq C^{\prime}(1+|x|)^{-c} .
$$

Thus, we obtain the algebraic decay rates of strong solutions to the Eq. (1.3). A corresponding result on algebraic decay rates of strong solutions for the CH equation can be found in [29].

(2) Choose $\phi=\phi_{a, 1,0,0}$ if $x \geq 0$, and $\phi(x)=1$ if $x \leq 0$ with $0 \leq a<1$. It is easy to see that such weight satisfies the admissibility conditions of Definition 4.1. Let further $p=\infty$ in Theorem 4.1, Then one deduces that Eq. (1.3) preserve the pointwise decay $O\left(e^{-a x}\right)$ as $x \rightarrow+\infty$ for any $t>0$. Similarly, we have persistence of the decay $O\left(e^{-a x}\right)$ as $x \rightarrow-\infty$. Theorem 4.1 thus generalizes the main result of [24, 26] on persistence of strong solutions to the CH type equations. It is worthwhile to note that Yin et al. also use weight functions and Gronwall's Lemma to obtain the exponential decay [[24], Theorem 5.1], in which they require the Sobolev index $s>5 / 2$, here we extend to $s>3 / 2$.

(3) Our result generalize the work of [3] on persistence and non-persistence of solutions to its supersymmetric extension. Meanwhile, since the dispersive term present in Eq. (1.3), we improved the corresponding result [[3], Theorem 2.2] to $p \in[1, \infty]$.

Clearly, the limit case $\phi=\phi_{1,1, c, d}$ is not covered by Theorem 4.1. In the following theorem however we may choose the weight $\phi=\phi_{1,1, c, d}$ with $c<0, d \in \mathbb{R}$, and $\frac{1}{|c|}<p \leq \infty$, or more generally when $(1+|\cdot|)^{c} \log (e+|\cdot|)^{d} \in L^{p}(\mathbb{R})$. See Corollary 4.1 below:

Corollary 4.1. Let $1 \leq p \leq \infty$ and $\phi$ be a v-moderate weight function as in Definition 4.1 satisfying

$$
v e^{-|\cdot|} \in L^{p}(\mathbb{R})
$$


Assume that $u_{0} \phi, u_{0, x} \phi, \rho_{0} \phi \in L^{p}(\mathbb{R})$ and $u_{0} \phi^{1 / 2}, u_{0, x} \phi^{1 / 2}, \rho_{0} \phi^{1 / 2} \in L^{2}(\mathbb{R})$. Let also $z=(u, \rho) \in \mathcal{C}\left([0, T), H^{s}(\mathbb{R})\right) \times$ $\mathcal{C}\left([0, T), H^{s-1}(\mathbb{R})\right), s>3 / 2$ be the strong solution of the Cauchy problem for Equation (1.3) emanating from $z_{0}=\left(u_{0}, \rho_{0}\right)$. Then,

$$
\sup _{t \in[0, T)}\left(\|u(t) \phi\|_{L^{p}}+\left\|u_{x}(t) \phi\right\|_{L^{p}}+\|\rho(t) \phi\|_{L^{p}}\right)
$$

and

$$
\sup _{t \in[0, T)}\left(\left\|u(t) \phi^{\frac{1}{2}}\right\|_{L^{2}}+\left\|u_{x}(t) \phi^{\frac{1}{2}}\right\|_{L^{2}}+\left\|\rho(t) \phi^{\frac{1}{2}}\right\|_{L^{2}}\right)
$$

are finite.

Remark 4.2. For the particular choice $c=d=0$ and $p=\infty$, we conclude from $\left|u_{0}(x)\right|+\left|u_{0, x}(x)\right|+\left|\rho_{0}(x)\right| \leq$ $C e^{-|x|}$ for any $x \in \mathbb{R}$ that the unique solution $z=(u, \rho) \in \mathcal{C}\left([0, T) ; H^{s}(\mathbb{R})\right) \times \mathcal{C}\left([0, T) ; H^{s-1}(\mathbb{R})\right)$ of $(1.3)$ with $z_{0}=\left(u_{0}, \rho_{0}\right)$ satisfies

$$
|u(x, t)|+\left|\partial_{x} u(x, t)\right|+|\rho(x, t)| \leq C^{\prime} e^{-|x|}
$$

uniformly in $\mathbb{R} \times[0, T)$.

First, we present some standard definitions. In general a weight function is simply a non-negative function. A weight function $v: \mathbb{R}^{n} \rightarrow \mathbb{R}$ is called sub-multiplicative if

$$
v(x+y) \leq v(x) v(y), \text { for all } x, y \in \mathbb{R}^{n} .
$$

Given a sub-multiplicative function $v$, a positive function $\phi$ is $v$-moderate if and only if

$$
\exists C_{0}>0: \phi(x+y) \leq C_{0} v(x) \phi(y), \text { for all } x, y \in \mathbb{R}^{n}
$$

If $\phi$ is $v$-moderate for some sub-multiplicative function $v$, then we say that $\phi$ is moderate. This is the usual terminology in time-frequency analysis papers [3]. Let us recall the most standard examples of such weights. Let

$$
\phi(x)=\phi_{a, b, c, d}(x)=e^{a|x|^{b}}(1+|x|)^{c} \log (e+|x|)^{d} .
$$

We have (see [3]) the following conditions:

(i) For $a, c, d \geq 0$ and $0 \leq b \leq 1$ such weight is sub-multiplicative.

(ii) If $a, c, d \in \mathbb{R}$ and $0 \leq b \leq 1$, then $\phi$ is moderate. More precisely, $\phi_{a, b, c, d}$ is $\phi_{\alpha, \beta, \gamma, \delta}$-moderate for $|a| \leq \alpha,|b| \leq \beta,|c| \leq \gamma$ and $|d| \leq \delta$

The elementary properties of sub-multiplicative and moderate weights can be find in [3]. Now, we prove Theorem 4.1.

Proof of Theorem 4.1. Let $z=(u, \rho)$ be the solution to Eq. (1.3) with initial data $z_{0} \in H^{s} \times H^{s-1}, s>3 / 2$, and $T$ be the maximal existence times of the solution $z$, which is guaranteed by Theorem 3.2 in [24].

From the solution $z=(u, \rho) \in \mathcal{C}\left([0, T), H^{s}(\mathbb{R})\right) \times \mathcal{C}\left([0, T), H^{s-1}(\mathbb{R})\right), s>3 / 2$, The Sobolev's imbedding theorem yields

$$
M \equiv \sup _{t \in[0, T]}\left(\|u(t, \cdot)\|_{L^{\infty}}+\left\|u_{x}(t, \cdot)\right\|_{L^{\infty}}+\|\rho(t, \cdot)\|_{L^{\infty}}\right)<\infty
$$


For any $N \in \mathbb{Z}^{+}$, let us consider the $N$-truncations of $\phi(x): f(x)=f_{N}(x)=\min \{\phi(x), N\}$. Then $f: \mathbb{R} \rightarrow \mathbb{R}$ is a locally absolutely continuous function such that

$$
\|f\|_{\infty} \leq N, \quad\left|f^{\prime}(x)\right| \leq A|f(x)| \quad \text { a.e. on } \mathbb{R} .
$$

In addition, if $C_{1}=\max \left\{C_{0}, \alpha^{-1}\right\}$, where $\alpha=\inf _{x \in \mathbb{R}} v(x)>0$, then

$$
f(x+y) \leq C_{1} v(x) f(y), \quad \forall x, y \in \mathbb{R} .
$$

Moreover, as shown in [3], the $N$-truncations $f$ of a $v$-moderate weight $\phi$ are uniformly $v$-moderate with respect to $N$.

Similar to [24], we rewrite Eq. (1.3) as the following nonlocal form

$$
\begin{cases}u_{t}+u u_{x}=-\partial_{x} G * P(u, \rho), & t>0, x \in \mathbb{R}, \\ \rho_{t}+u \rho_{x}=-(b-1) u_{x} \rho, & t>0, x \in \mathbb{R}, \\ u(x, 0)=u_{0}(x), \rho(x, 0)=\rho_{0}(x), & x \in \mathbb{R},\end{cases}
$$

where $P(u, \rho) \doteq \frac{b}{2} u^{2}+\frac{3-b}{2} u_{x}^{2}+\frac{\kappa}{2} \rho^{2}-\alpha u$ and $G(x)=\frac{1}{2} e^{-|x|}$.

Let us start from the case $1 \leq p<\infty$. Multiplying the first equation in Eq. (4.4) by $|u f|^{p-1} \operatorname{sgn}(u f) f$ and integrating it lead to

$$
\int_{\mathbb{R}}|u f|^{p-1} \operatorname{sgn}(u f)\left(\partial_{t} u f\right) d x=\int_{\mathbb{R}}|u f|^{p-1} \operatorname{sgn}(u f) f u u_{x} d x-\int_{\mathbb{R}}|u f|^{p-1} \operatorname{sgn}(u f) f \cdot \partial_{x}(G * P(u, \rho)) d x,
$$

The first term on the left hand of (4.5) reads

$$
\int_{\mathbb{R}}|u f|^{p-1} \operatorname{sgn}(u f)\left(\partial_{t} u f\right) d x=\frac{1}{p} \frac{d}{d t}\|u f\|_{L^{p}}^{p}=\|u f\|_{L^{p}}^{p-1} \frac{d}{d t}\|u f\|_{L^{p}} .
$$

Then, the Hölder's inequality is followed by the estimate

$$
\left.\left|\int_{\mathbb{R}}\right| u f\right|^{p-1} \operatorname{sgn}(u f) u u_{x} f d x \mid \leq\|u f\|_{L^{p}}^{p-1}\left\|u u_{x} f\right\|_{L^{p}} \leq M\|u f\|_{L^{p}}^{p-1}\left\|u_{x} f\right\|_{L^{p}} .
$$

For the nonlocal term, we have

$$
\begin{aligned}
& \left.\left|\int_{\mathbb{R}}\right| u f\right|^{p-1} \operatorname{sgn}(u f)\left[f \cdot \partial_{x}(G * P(u, \rho))\right] d x \mid \leq\|u f\|_{L^{p}}^{p-1}\left\|f \cdot \partial_{x}(G * P(u, \rho))\right\|_{L^{p}} \\
& \quad \leq C_{\alpha, b, \kappa}\|u f\|_{L^{p}}^{p-1}\left\{\left\|\left(\partial_{x} G\right) v\right\|_{L^{1}}\left\|f \cdot\left(u+u^{2}+u_{x}^{2}+\rho^{2}\right)\right\|_{L^{p}}\right\} \\
& \quad \leq C(1+M)\|u f\|_{L^{p}}^{p-1}\left(\|u f\|_{L^{p}}+\left\|u_{x} f\right\|_{L^{p}}+\|\rho f\|_{L^{p}}\right),
\end{aligned}
$$

where the Hölder's inequality, Proposition 3.1 and 3.2 in [3], and Condition (4.1) are applied in the first inequality, the second one, and the last one, respectively, and the constant $C$ only depends on $v$ and $\phi$. Form Eq. (4.5) one may get

$$
\frac{d}{d t}\|u f\|_{L^{p}} \leq C(1+M)\left(\|u f\|_{L^{p}}+\left\|u_{x} f\right\|_{L^{p}}+\|\rho f\|_{L^{p}}\right) .
$$

Let us now give the estimate on $u_{x} f$. Differentiating the first equation in Eq. (4.4) with respect to the variable $x$ and then multiplying by $f$, we may arrive at

$$
\partial_{t}\left(u_{x} f\right)+u u_{x x} f+u_{x}^{2} f+f \partial_{x}^{2}(G * P(u, \rho))=0,
$$


which yields

$$
\begin{gathered}
\int_{\mathbb{R}}\left|u_{x} f\right|^{p-1} \operatorname{sgn}\left(u_{x} f\right) \partial_{t}\left(u_{x} f\right) d x=\left\|u_{x} f\right\|_{L^{p}}^{p-1} \frac{d}{d t}\left\|u_{x} f\right\|_{L^{p}} \\
\left.\left|\int_{\mathbb{R}}\right| u_{x} f\right|^{p-1} \operatorname{sgn}\left(u_{x} f\right) f u_{x}^{2} d x\left|\leq\left\|\left.u_{x} f\right|_{L^{p}} ^{p-1}\right\| u_{x} f u_{x}\left\|_{L^{p}} \leq M\right\| u_{x} f\left\|_{L^{p}}^{p-1}\right\| u_{x} f \|_{L^{p}}\right.
\end{gathered}
$$

and

$$
\begin{gathered}
\left.\left|\int_{\mathbb{R}}\right| u_{x} f\right|^{p-1} \operatorname{sgn}\left(u_{x} f\right)\left[f \partial_{x}^{2}(G * P(u, \rho))\right] d x \mid \leq\left\|u_{x} f\right\|_{L^{p}}^{p-1}\left\|f \partial_{x}^{2}(G * P(u, \rho))\right\|_{L^{p}} \\
\leq C(1+M)\left\|u_{x} f\right\|_{L^{p}}^{p-1}\left(\|u f\|_{L^{p}}+\left\|u_{x} f\right\|_{L^{p}}+\|\rho f\|_{L^{p}}\right) .
\end{gathered}
$$

For the second order derivative term, we have

$$
\begin{aligned}
& \left.\left|\int_{\mathbb{R}}\right| u_{x} f\right|^{p-1} \operatorname{sgn}\left(u_{x} f\right) u u_{x x} f d x \mid \\
& \left.\quad=\left.\left|\int_{\mathbb{R}}\right| u_{x} f\right|^{p-1} \operatorname{sgn}\left(u_{x} f\right) u\left[\partial_{x}\left(u_{x} f\right)-u_{x} f_{x}\right)\right] d x \mid \\
& \quad=\left.\left|\int_{\mathbb{R}} u \partial_{x}\left(\frac{\left|u_{x} f\right|^{p}}{p}\right) d x-\int_{\mathbb{R}}\right| u_{x} f\right|^{p-1} \operatorname{sgn}\left(u_{x} f\right) u u_{x} f_{x} d x \mid \\
& \quad \leq\left. M(1+A)|| u_{x} f\right|_{L^{p}} ^{p},
\end{aligned}
$$

where the inequality $\left|f_{x}(x)\right| \leq A f(x)$, for a.e. $x$, is applied. Thus, it follows that

$$
\frac{d}{d t}\left\|u_{x} f\right\|_{L^{p}} \leq C_{3}(1+M)\left(\|u f\|_{L^{p}}+\left\|u_{x} f\right\|_{L^{p}}+\|\rho f\|_{L^{p}}\right) .
$$

We now multiply the second equation in Eq. (4.4) with $|\rho f|^{p-1} \operatorname{sgn}(\rho f) f$ and integrate to obtain the identity

$$
\frac{1}{p} \frac{d}{d t}\|\rho f\|_{L^{p}}^{p}+\int_{\mathbb{R}}|\rho f|^{p-1} \operatorname{sgn}(\rho f) f u \rho_{x} d x+(b-1) \int_{\mathbb{R}}|\rho f|^{p-1} \operatorname{sgn}(\rho f) f u_{x} \rho d x=0 .
$$

As above, we get

$$
\left.\left|\int_{\mathbb{R}}\right| \rho f\right|^{p-1} \operatorname{sgn}(\rho f) f u_{x} \rho d x \mid \leq\|\rho f\|_{L^{p}}^{p-1}\left\|f u_{x} \rho\right\|_{L^{p}} \leq M\|\rho f\|_{L^{p}}^{p-1}\|\rho f\|_{L^{p}}
$$

and

$$
\begin{aligned}
\left.\left|\int_{\mathbb{R}}\right| \rho f\right|^{p-1} \operatorname{sgn}(\rho f) f u \rho_{x} d x \mid & \left.=\left.\left|\int_{\mathbb{R}}\right| \rho f\right|^{p-1} \operatorname{sgn}(\rho f) u\left[\partial_{x}(\rho f)-\rho f_{x}\right)\right] d x \mid \\
& =\left.\left|\int_{\mathbb{R}} u \partial_{x}\left(\frac{|\rho f|^{p}}{p}\right) d x-\int_{\mathbb{R}}\right| \rho f\right|^{p-1} \operatorname{sgn}(\rho f) u \rho f_{x} d x \mid \\
& \leq\left. M(1+A)|| \rho f\right|_{L^{p}} ^{p},
\end{aligned}
$$

this yields

$$
\frac{d}{d t}\|\rho f\|_{L^{p}} \leq C_{4} M\|\rho f\|_{L^{p}}
$$

Based on the inequalities (4.6)-(4.8), by Gronwall's inequality

$\|u(t) f\|_{L^{p}}+\left\|u_{x}(t) f\right\|_{L^{p}}+\|\rho(t) f\|_{L^{p}} \leq\left(\left\|u_{0} f\right\|_{L^{p}}+\left\|u_{0, x} f\right\|_{L^{p}}+\left\|\rho_{0} f\right\|_{L^{p}}\right) \exp (C(1+M) t)$, for all $t \in[0, T)$.

Since $f(x)=f_{N}(x) \uparrow \phi(x)$ as $N \rightarrow \infty$ for a.e. $x \in \mathbb{R}$ and $u_{0} \phi, u_{0, x} \phi, \rho_{0} \phi \in L^{p}(\mathbb{R})$ the assertion of the theorem follows for the case $p \in[1, \infty)$. Since $\|\cdot\|_{L^{\infty}}=\lim _{p \rightarrow \infty}\|\cdot\|_{L^{p}}$ it is clear that the theorem also applies for $p=\infty$. 
Proof of Corollary 4.1. As explained in [3], if the function $\phi$ is a $v$-moderate weight function, then the function $\phi^{1 / 2}$ is also a $v^{1 / 2}$-moderate weight satisfying $\left|\left(\phi^{1 / 2}\right)^{\prime}(x)\right| \leq \frac{A}{2} \phi^{1 / 2}(x)$, inf $v^{1 / 2}>0$ and $v^{1 / 2} e^{-|\cdot|} \in$ $L^{1}(\mathbb{R})$. We use Theorem 4.1 with $p=2$ to the weight $\phi^{1 / 2}$ and obtain

$$
\left\|u(t) \phi^{\frac{1}{2}}\right\|_{L^{2}}+\left\|u_{x}(t) \phi^{\frac{1}{2}}\right\|_{L^{2}}+\left\|\rho(t) \phi^{\frac{1}{2}}\right\|_{L^{2}} \leq\left(\left\|u_{0} \phi^{\frac{1}{2}}\right\|_{L^{2}}+\left\|u_{0, x} \phi^{\frac{1}{2}}\right\|_{L^{2}}+\left\|\rho_{0} \phi^{\frac{1}{2}}\right\|_{L^{2}}\right) \exp (C(1+M) t) .
$$

In view of Proposition 3.2 in [3], noticing $f(x)=f_{N}(x)=\min \{\phi(x), N\}$ admits

$$
\begin{aligned}
& \left\|f \partial_{x}\left(G * P\left(u, u_{x}\right)\right)\right\|_{L^{p}}\left\|_{L^{p}} \leq C_{\alpha, \kappa, b}\right\| f \partial_{x}\left(G(x) *\left(u+u^{2}+u_{x}^{2}+\rho^{2}\right)\right) \|_{L^{p}} \\
& \quad \leq C\left\|f \partial_{x} G(x)\right\|_{L^{p}}\left\|f\left(u+u^{2}+u^{3}+u^{4}+u_{x}^{2}\right)\right\|_{L^{1}} \\
& \quad \leq C\left\|f e^{-|x|}\right\|_{L^{p}}\left(\|f u\|_{L^{1}}+\left\|f^{\frac{1}{2}} u\right\|_{L^{2}}^{2}+\left\|f^{\frac{1}{2}} u_{x}\right\|_{L^{2}}^{2}+\left\|f^{\frac{1}{2}} \rho\right\|_{L^{2}}^{2}\right) \\
& \quad \leq C_{1} \exp \left(C_{2}(1+M) t\right),
\end{aligned}
$$

where we used (4.9) and Theorem 4.1 with $p=1$.

Similarly, noticing $\partial_{x}^{2} G=G-\delta$ reveals

$$
\left\|f \partial_{x}^{2}\left(G * P\left(u, u_{x}\right)\right)\right\|_{L^{p}} \leq C_{1} \exp \left(C_{2}(1+M) t\right)+C_{3}(1+M)\left(\|u f\|_{L^{p}}+\left\|f u_{x}\right\|_{L^{p}}+\|f \rho\|_{L^{p}}\right)
$$

where the constants on the right-hand side of Eqs. (4.10) and (4.11) are independent of $N$.

By using the procedure as shown in the proof of Theorem 4.1, we can readily obtain

$$
\frac{d}{d t}\|u f\|_{L^{p}} \leq C(1+M)\|u f\|_{L^{p}}+\left\|f \partial_{x}\left(G * G\left(u, u_{x}\right)\right)\right\|_{L^{p}}, \text { for } 1 \leq p<\infty,
$$

and

$$
\frac{d}{d t}\left\|u_{x} f\right\|_{L^{p}} \leq C(1+M)\left\|u_{x} f\right\|_{L^{p}}+\left\|f \partial_{x}^{2}\left(G * P\left(u, u_{x}\right)\right)\right\|_{L^{p}}, \text { for } 1 \leq p<\infty
$$

Plugging Eqs. (4.10) and (4.11) into Eqs. (4.12) and (4.13), respectively, and summing up them, we obtain

$$
\begin{aligned}
\frac{d}{d t}\left(\|u(t) f\|_{L^{p}}+\left\|u_{x}(t) f\right\|_{L^{p}}+\|\rho(t) f\|_{L^{p}}\right) \leq & K_{1}(1+M)\left(\left\|u_{0} f\right\|_{L^{p}}+\left\|u_{0, x} f\right\|_{L^{p}}+\left\|\rho_{0} f\right\|_{L^{p}}\right) \\
& +C_{1} \exp \left(C_{2}(1+M) t\right),
\end{aligned}
$$

which is taken integration and limit $N \rightarrow \infty$ to get the conclusion in the case $1 \leq p<\infty$. The constants throughout the proof are independent of $p$. Therefore, for $p=\infty$ one can rely on the result established for the finite exponents $q$ and then let $q \rightarrow \infty$. The rest of the theorem is fully similar to that of Theorem 4.1.

\section{Acknowledgments}

The authors are very grateful to the anonymous reviewers for their careful read and useful suggestions, which greatly improved the presentation of the paper.

\section{References}

[1] V. I. Arnold, Sur la géométrie différentielle des groupes de Lie de dimension infinie et ses applications àl' hydrodynamique des fluides parfaits, Ann. Inst. Fourier (Grenoble), 16 (1966), 319-361. 
[2] H. Bahouri, J. Y. Chemin and R. Danchin, Fourier Analysis and Nonlinear Partial Differential Equations, Springer-Verlag, Berlin, Heidelberg, 2011.

[3] L. Brandolese, Breakdown for the Camassa-Holm equation using decay criteria and persistence in weighted spaces, Int. Math. Res. Not. 22 (2012), 5161-5181.

[4] R. Camassa and D. Holm, An integrable shallow water equation with peaked solitons, Phys. Rev. Letters 71 (1993), 1661-1664.

[5] R. Camassa, D. Holm and J. Hyman, A new integrable shallow water equation, Adv. Appl. Mech. 31 (1994), 1-33.

[6] G. M. Coclite, H. Holden and K. H. Karlsen, Well-posedness of higher-order Camassa-Holm equations, J. Differ. Eq. 246 (2009), 929-963.

[7] A. Constantin, Existence of permanent and breaking waves for a shallow water equation: a geometric approach, Ann. Inst. Fourier (Grenoble), 50 (2000), 321-362.

[8] A. Constantin and J. Escher, Wave breaking for nonlinear nonlocal shallow water equations, Acta Math. 181 (1998), 229-243.

[9] A. Constantin and J. Escher, Well-posedness, global existence and blow-up phenomena for a periodic quasi-linear hyperbolic equation, Comm. Pure Appl. Math. 51 (1998), 475-504.

[10] A. Constantin and R. Ivanov, On an integrable two-component Camassa-Holm shallow water system, Phys. Lett. A, 372 (2008), 7129-7132.

[11] A. Constantin and B. Kolev, Geodesic flow on the diffeomorphism group of the circle, Comment. Math. Helv. 78 (2003), $787-804$.

[12] R. Danchin, A note on well-posedness for Camassa-Holm equation, J. Differential Equations 192 (2003), 429-444.

[13] R. Danchin, A few remarks on the Camassa-Holm equation, Differential Integral Equations 14 (2001), 953-988.

[14] A. Degasperis and M. Procesi, Asymptotic integrability, in: Symmetry and Perturbation Theory, World Scientific, Singapore, 1999, pp. 23-37.

[15] H. R. Dullin, G. A. Gottwald and D. D. Holm, Camassa-Holm, Korteweg-de Vries-5 and other asymptotically equivalent equations for shallow water waves, Fluid Dyn. Res. 33 (2003), 73-79.

[16] H. R. Dullin, G. A. Gottwald and D. D. Holm, On asymptotically equivalent shallow water wave equations, Phys. D. 190 (2004), 1-14.

[17] H. R. Dullin, G. A. Gottwald and D. D. Holm, An integrable shallow water equation with linear and nonlinear dispersion, Phys. Rev. Letters 87 (2001), 4501-4504.

[18] D. G. Ebin and J. E. Marsden, Groups of diffeomorphisms and the motion of an incompressible fluid, Ann. Math. 92 (1970), 102-163.

[19] J. Escher, D. Henry, B. Kolev and T. Lyons, Two-component equations modelling water waves with constant vorticity, Ann. Mat. Pura Appl. 195 (2016), 249-271.

[20] J. Escher and T. Lyons, Two-component higher order Camassa-Holm systems with fractional inertia operator: A geometric approach, J. Geom. Mech. 7 (2015), 281-293.

[21] J. Escher and Z. Yin, Well-posedness, blow-up phenomena, and global solutions for the b-equation, J. reine Angew. Math. 624 (2008), 51-80.

[22] J. Escher, and B. Kolev, The Degasperis-Procesi equation as a non-metric Euler equation, Math. Z. 269 (2011), $1137-1153$. 
[23] J. Escher and B. Kolev, Right-invariant Sobolev metrics of fractional order on the diffeomorphism group of the circle, J. Geom. Mech. 6 (2014), 335-372.

[24] C. Guan, H. He and Z. Yin, Well-posedness, blow-up phenomena and persistence properties for a two-component water wave system, Nonlinear Anal. Real World Appl. 25 (2015) 219-237.

[25] D. Henry, Compactly supported solutions of a family of nonlinear partial differential equations, Dyn. Contin. Discrete Impuls. Syst. Ser. A Math. Anal. 15 (2008), 145-150.

[26] A. A. Himonas, G. Misiolek, G. Ponce and Y. Zhou, Persistence properties and unique continuation of solutions of the Camassa-Holm equation, Comm. Math. Phy. 271 (2007), 511-522.

[27] R. McLachlan and X. Zhang, Well-posedness of modified Camassa-Holm equations, J. Diff. Eq. 246(2009), 3241-3259.

[28] G. Misiołek, Classical solutions of the periodic Camassa-Holm equation, Geom. Funct. Anal. 12 (2002), $1080-1104$.

[29] L. D. Ni and Y. Zhou, A new asymptotic behavior of solutions to the Camassa-holm equation, Proc. Amer. Math. Soc. 140 (2012), 607-614.

[30] Z. Popowicz, A two-component generalization of the Degasperis-Procesi equation, J. Phys. A 39 (2006), 13717-13726.

[31] S. Zhou, Persistence properties for a generalized Camassa-Holm equation in weighted $L^{p}$ spaces, J. Math. Anal. Appl. 410 (2014), 932-938.

[32] S. Zhou and C. Mu, The properties of solutions for a generalized b-family equation with higher-order nonlinearities and peakons, J. Nonlinear Sci. 23 (2013), 863-889.

[33] S. Zhou, C. Mu and R. Zeng Well-posedness and blow-up phenomena for a higher order shallow water equation, J. Differential Equations 251 (2011), 3488-3499.

[34] S. Zhou, C. Mu and L. Wang, Well-posedness, blow-up phenomena and global existence for the generalized b-equation with higher-order nonlinearities and weak dissipation, Discrete Contin. Dyn. Syst. 32 (2014), 843-867. 